\title{
Canonical triangulations of Dehn fillings
}

\author{
FRANÇOIS GUÉRITAUD \\ SAUL SCHLEIMER
}

\begin{abstract}
Every cusped, finite-volume hyperbolic three-manifold has a canonical decomposition into ideal polyhedra. We study the canonical decomposition of the hyperbolic manifold obtained by filling some (but not all) of the cusps with solid tori: in a broad range of cases, generic in an appropriate sense, this decomposition can be predicted from that of the unfilled manifold (a similar result has been independently announced by Akiyoshi [4]). We also find the canonical decompositions of all hyperbolic Dehn fillings on one cusp of the Whitehead link complement.
\end{abstract}

51H20; 57M50

\section{Introduction}

Let $M$ be a complete cusped hyperbolic 3-manifold of finite volume, and endow the cusps $c_{1}, \ldots, c_{k}$ of $M$ with disjoint simple horoball neighborhoods $H_{1}, \ldots, H_{k}$. The Ford-Voronoi domain $\mathcal{F} \subset M$ consists of all points of $M$ having a unique shortest path to the union of the $H_{i}$. The complement of $\mathcal{F}$ is a compact complex $C$ of totally geodesic polygons. By definition, the canonical decomposition $\mathcal{D}$ of $M$ with respect to the $H_{i}$ has one 3-dimensional cell (an ideal polyhedron) per vertex of $C$, one face per edge of $C$, and one edge per (polygonal) face of $C$; we say that $\mathcal{D}$ is dual to $C$. Other names for $\mathcal{D}$ are the geometrically canonical decomposition, or Delaunay (or Delone) decomposition. In [11], Epstein and Penner give a precise description of $\mathcal{D}$ in terms of convex hulls in Minkowski space $\mathbb{R}^{3+1}$. Weeks' program SnapPea [27] will compute $\mathcal{D}$ for most manifolds of moderate size.

Akiyoshi [3] proves that, as the volumes of $\left\{H_{i}\right\}_{1 \leq i \leq k}$ vary, only finitely many decompositions $\mathcal{D}$ arise. By Mostow-Prasad rigidity, the resulting collection of Delaunay decompositions is a complete topological invariant of $M$. When $M$ has a single cusp there is a unique Delaunay decomposition. When $M$ has multiple cusps one may take all of their volumes to be equal; SnapPea uses the resulting decomposition for rigorous computation of isometry groups and detection of isometric manifolds (see Weeks [28]).

Thus canonical decompositions lead to an interplay between hyperbolic geometry and the combinatorics of cell-decompositions. This motivates the study of $\mathcal{D}$ and 
suggests that it is a difficult problem in general. General results are known only when $M$ is restricted to belong to certain classes of manifolds: punctured-torus bundles, two-bridge link complements, certain arborescent link complements and related objects, or covers of any of these spaces - see Jørgensen [19], Akiyoshi [2], Lackenby [20], Akiyoshi et al [6; 7] and Guéritaud [15; 16; 14]. In fact, the combinatorics underlying all the above examples are to a large extent the same. More examples, often using symmetry, are compiled by Sakuma and Weeks [25].

The present paper offers a relative result: we are interested in how the canonical decomposition $\mathcal{D}$ changes when the last cusp $c_{k}$ (where $k \geq 2$ ) undergoes a Dehn filling along the slope $s$. Recall the operation of filling along $s$ removes the interior of $H_{k}$ from $M$ and glues a solid torus $X_{s}$ to the resulting boundary component, yielding the filled manifold $M_{s}$. Thurston showed that the metric on $M_{s}$ Gromovconverges, with appropriate choices of basepoints, to the metric on $M$ as the length of the filling slope $s$ goes to infinity. Consequently, a Margulis tube (region where the injectivity radius is less than the Margulis constant) appears about the core curve of $X_{S}$ [26].

Experimentation with SnapPea suggests that, for many manifolds, cusps, and slopes, after filling $c_{k}$ the polyhedra of $\mathcal{D}$ outside the Margulis tube undergo only a small geometric perturbation while the combinatorics of $\mathcal{D}_{s}$ inside the tube has a predictable structure. To ensure such good behavior we choose the reference horoball neighborhoods $\left\{H_{i}\right\}_{1 \leq i<k}$ of the remaining cusps after filling to have the same volumes as before filling. Moreover, we take the horoball neighborhood $H_{k}$ before filling to be very small and we make two "genericity" assumptions:

(I) The decomposition $\mathcal{D}$ (before filling) consists only of ideal tetrahedra.

(II) There exists a unique shortest path from $H_{k}$ to $\bigcup_{i=1}^{k-1} H_{i}$ in $M$.

Of course, this notion of genericity is problematic as there are only countably many complete finite-volume cusped hyperbolic 3-manifolds; infinitely many of these are nongeneric. Still, SnapPea verifies that of the fifteen twice-cusped manifolds from the five-tetrahedron census (see Callahan, Hildebrand and Weeks [8]) (m125, m129, m202, m203, m292, m295, m328, m329, m357, m359, m366, m367, m388, m391, $\mathrm{m} 412$ ), eleven are generic. The Whitehead sister m125 fails (I), while the Whitehead link m129, as well as m203 and m412, fail both (I) and (II). All 15 admit involutions switching the cusps. All 15 except $\mathrm{m} 412$ are obtained by filling a cusp of the census manifold s776, itself generic (the "magic manifold" of Martelli and Petronio [21]).

It is a corollary of Theorem 1 that if a generic manifold $M$ has $k$ cusps, if the horoball $H_{k-1}$, like $H_{k}$, has a unique shortest path to $H_{1} \cup \cdots \cup H_{k-2}$, and if both 
$H_{k-1}$ and $H_{k}$ are small, then all but finitely many fillings on $c_{k}$ are again generic with respect to $c_{k-1}$. In short, almost all fillings of generic manifolds (such as s776) are again generic.

Theorem 1 Under the genericity assumptions (I)-(II) above, if the volume of the cusp neighborhood $H_{k}$ is small enough, then the decomposition $\mathcal{D}$ (before filling) contains exactly two ideal tetrahedra $\Delta, \Delta^{\prime}$ that have a vertex in the cusp $c_{k}$. The tetrahedra $\Delta, \Delta^{\prime}$ are isometric, each of $\Delta, \Delta^{\prime}$ has exactly one vertex in $c_{k}$, and $\partial\left(\Delta \cup \Delta^{\prime}\right)$ is a once-punctured torus. For all but finitely many filling slopes $s$ in the cusp $c_{k}$, the canonical decomposition $\mathcal{D}_{s}$ of the manifold obtained by Dehn filling along $s$ is combinatorially of the form

$$
\mathcal{D}_{s}=\left(\mathcal{D} \backslash\left\{\Delta, \Delta^{\prime}\right\}\right) \cup \mathcal{T}
$$

where $\mathcal{T}=\left\{\Delta_{1}, \ldots, \Delta_{N}\right\}$ is a triangulation of a solid torus minus one boundary point, and the combinatorial gluing of the $\Delta_{i}$ is dictated by the continued fraction expansion of the slope $s$, with respect to a certain basis of the first homology of the cusp $c_{k}$ depending only on $\mathcal{D}$.

As set out in Section 2, the combinatorics of the triangulation $\mathcal{T}$ are identical to a procedure found in the SnapPea kernel [27], called the layering construction by Jaco and Rubinstein [17]. Each integer $\alpha$ near the middle of the continued fraction expansion gives rise to $\alpha$ adjacent tetrahedra, to one edge of degree $2 \alpha+4$, and to $\alpha-1$ edges of degree 4 (the average degree of edges is always 6 by an Euler characteristic argument). See Section 2 for details.

Geometrically, the tetrahedra of $\mathcal{D}_{s} \backslash \mathcal{T}$ are small deformations of the tetrahedra of $\mathcal{D} \backslash\left\{\Delta \cup \Delta^{\prime}\right\}$. To predict $\mathcal{D}_{s}$ when genericity is not satisfied, or even to estimate the number of slopes $s$ which fail to be sufficiently large in the sense of Theorem 1 (their number may not be universally bounded), remains very challenging.

We will prove Theorem 1 in Section 4. Moreover, an analogous statement (Theorem 26) will still hold when more than one cusp is filled. In Section 5, we will treat a real-life family of examples by showing:

Theorem 2 If $M$ is a hyperbolic Dehn filling of one cusp of the Whitehead link complement in $\mathbb{S}^{3}$, the canonical decomposition of $M$ is dictated by the continued fraction expansion of the filling slope.

The Whitehead link complement actually violates both conditions (I)-(II) of the genericity assumption, but its symmetry makes up for this inconvenience. In fact, we will 
construct a certain triangulated solid torus, also denoted $\mathcal{T}$, that serves as a proxy for the Margulis (filling) tube: in the case of the Whitehead link complement, it turns out that the filled manifold consists only of $\mathcal{T}$ with some exterior faces pairwise identified, ie no combinatorics outside $\mathcal{T}$ need to be remembered from the unfilled manifold. However, $\mathcal{T}$ can be slightly more complicated than in Theorem $1-$ see Section 5 for details.

Historically, the first avatar of the triangulation $\mathcal{T}$ of Theorem 1 seems to go back to [18] where Jørgensen briefly described the Ford-Voronoi domain of the quotient of $\mathbb{H}^{3}$ by a loxodromy, with respect to an ideal point $p$. Full proofs of his results were given by Drumm and Poritz [10], who also allow $p$ to be nonideal. For ideal $p$, we use angle structures and ideal triangulations (combinatorially dual to the Ford-Voronoi domain) to obtain new and quite different proofs of these results. Additionally, this technique provides the following improvements over the existing literature:

- Suppose that $\Gamma$ is a Kleinian group and $Z \subset \Gamma$ an infinite cyclic subgroup resulting from a Dehn filling. Then the canonically triangulated solid torus corresponding to $Z$ (provided, say, by [10]) is incorporated into the canonical triangulation of $\mathbb{H}^{3} / \Gamma$. Under the genericity assumption this incorporation explains how, in the program SnapPea, the picture of a triangulated cusp neighborhood changes under Dehn filling.

- In Section 5.4, we sketch an extension to the case where $Z$ is only virtually cyclic.

- The convex hull in $\mathbb{H}^{3}$ of an ideal loxodromic orbit always admits a canonical triangulation by the Epstein-Penner construction (extended to the infinitecovolume case by Akiyoshi and Sakuma [5]). However, some of the outermost tetrahedra may be timelike or lightlike, and not spacelike, in which case they do not correspond to vertices of the Ford-Voronoi domain (which indeed may have no vertices at all, eg for loxodromies with very small rotation number). Although this case does not arise in the context of Dehn fillings because the covolume stays finite [11], it is covered at no extra cost by our methods, and apparently eludes those of [10].

After this paper was accepted, M Sakuma pointed out to us the research report [4] where Akiyoshi announced a result roughly equivalent to Theorem 1, sketching an argument more in line with Jørgensen's original approach. Earlier, using numerical methods, he also established [1] that the canonical decompositions of hyperbolic fillings of one cusp of the Whitehead link complement contain only tetrahedra, a consequence of Theorem 2 here. 
The plan of the paper is as follows. In Section 1 we recall the definition of the space $W$ of angle structures on a combinatorial ideal triangulation, and explain (following Rivin [23]) how to find the hyperbolic structure by maximizing a volume functional $\mathcal{V}$ on $W$; as an application we prove a rigidity result for solid tori. In Section 2 we recall the combinatorics of the Farey graph in $\mathbb{H}^{2}$ and use it to describe an ideal triangulation of a solid torus $\mathcal{T}$. In Section 4, using results from [16], we check that the decomposition of $\mathcal{T}$ is geometrically canonical, and describe how to insert $\mathcal{T}$ as a proxy Margulis tube of a filled manifold, under the genericity assumptions (I)-(II). In Section 5, we adapt the method to treat all Dehn fillings on one component of the Whitehead link complement.

Acknowledgements We are very grateful to PCMI (Park City), where this work originated during the summer of 2006. This project would have been impossible without Jeff Weeks' program SnapPea [27].

This work is in the public domain.

\section{Angle structures and volume maximization}

In Section 1.1 we give basic definitions and quote Theorem 5 (due to Rivin), the cornerstone of our method to find positively oriented ideal triangulations. In Section 1.2, we parametrize the deformation space of certain hyperbolic solid tori; the method, while not a direct application of Theorem 5, follows from the same ideas and from the concept of "spun" triangulations [26].

\subsection{Rivin's theorem}

Definition 3 A (combinatorial) ideal tetrahedron is a space diffeomorphic to an ideal tetrahedron of hyperbolic space $\mathbb{H}^{3}$ (ie with vertices at infinity); the faces of such an ideal tetrahedron are called ideal triangles.

Consider an oriented combinatorial ideal tetrahedron $\Delta$, and copies $\Delta_{1}, \ldots, \Delta_{N}$ of $\Delta$ : the $\partial \Delta_{i}$ naturally receive consistent orientations. A gluing of the $\Delta_{i}$ is an equivalence relation on $\bigsqcup_{i=1}^{N} \Delta_{i}$ generated by orientation-reversing identifications $\phi_{F G}: G \rightarrow F$ of pairs of faces $F \neq G$ of the $\Delta_{i}$, in such a way that:

- For each face $F$ of each $\Delta_{i}$, there is at most one face $G$ (resp. $H$ ) of some $\Delta_{j}$ such that $\varphi_{F G}$ (resp. $\varphi_{H F}$ ) is defined; moreover $G$ exists if and only if $H$ exists and one then has $G=H$ and $\varphi_{H F}=\varphi_{F G}^{-1}$. 
- Whenever $\varphi:=\varphi_{F_{1} F_{2}} \circ \varphi_{F_{2} F_{3}} \circ \cdots \circ \varphi_{F_{n-1} F_{n}} \circ \varphi_{F_{n} F_{1}}$ is well-defined on an edge $\epsilon$ of $\Delta_{i}$, then $\varphi$ is the identity on $\epsilon$.

The last condition is called the trivial holonomy condition.

Let $\sim$ be a gluing. Then $M:=\left(\bigsqcup_{i=1}^{N} \Delta_{i}\right) / \sim$ is a manifold (possibly with boundary). We say that the $\Delta_{i}$ endow $M$ with an ideal triangulation. The $6 N$ edges of the $\Delta_{i}$ define edges in $M$, which we call boundary edges if they belong to $\partial M$, and interior edges otherwise.

Let us denote by $\epsilon_{i}^{1}, \ldots, \epsilon_{i}^{6}$ the six edges of $\Delta_{i}$ (before gluing), and by $E$ the set of all $\epsilon_{i}^{\kappa}$ (so $|E|=6 N$ ). We say that $\epsilon \in E$ is incident to an edge $e$ of $M$ if $\epsilon$ projects to $e$ under the gluing “ $\sim$ ". Fix a map $\alpha$ : $\{$ boundary edges of $M\} \rightarrow \mathbb{R}^{+}$.

Definition 4 An angle structure on $M$ with respect to $\alpha$ is a map $\theta: E \rightarrow \mathbb{R}_{+}^{*}$ such that

- if the edges $\epsilon, \epsilon^{\prime}, \epsilon^{\prime \prime}$ of $\Delta_{i}$ share a vertex, then $\theta(\epsilon)+\theta\left(\epsilon^{\prime}\right)+\theta\left(\epsilon^{\prime \prime}\right)=\pi$;

- if $\epsilon_{1}, \ldots, \epsilon_{n} \in E$ is the full list of edges incident to an interior edge $e$ of $M$, then $\sum_{i=1}^{n} \theta\left(\epsilon_{i}\right)=2 \pi$;

- if $\epsilon_{1}, \ldots, \epsilon_{n} \in E$ is the full list of edges incident to a boundary edge $e$ of $M$, then $\sum_{i=1}^{n} \theta\left(\epsilon_{i}\right)=\pi-\alpha(e)$. (This is a convexity condition on $M$, since $\alpha \geq 0$.)

The $\theta(\epsilon)$, for $\epsilon \in E$, are called the dihedral angles of the $\Delta_{i}$. Note that in an angle structure, the dihedral angles at opposite edges of any tetrahedron $\Delta_{i}$ are equal. Thus we can realize each $\Delta_{i}$ by an ideal hyperbolic tetrahedron $\delta_{i}$ of $\mathbb{H}^{3}$ with dihedral angles $\theta\left(\epsilon_{i}^{1}\right), \ldots, \theta\left(\epsilon_{i}^{6}\right)$; likewise, the total dihedral angles about every interior edge is $2 \pi$. However, when the face identifications $\varphi_{F G}$ are the corresponding hyperbolic isometries, the trivial holonomy condition may be violated. The following theorem tells us exactly for which angle structures this problem disappears. Let $\Lambda$ be the Lobachevski function defined by $\Lambda(x):=-\int_{0}^{x} \log |2 \sin t| d t$.

Theorem 5 (Rivin [23]) Suppose the space $W$ of angle structures is nonempty. Then every critical point $\theta \in W$ of the volume functional

$$
\mathcal{V}(\theta):=\frac{1}{2} \sum_{\epsilon \in E} \Lambda(\theta(\epsilon))>0
$$

defines a complete hyperbolic metric with polyhedral boundary on $M$, with exterior dihedral angle $\alpha(e)$ at each exterior edge $e$. Conversely, if $M$ admits such a complete hyperbolic metric in which the $\Delta_{i}$ are realized by totally geodesic ideal tetrahedra $\delta_{i}$ with disjoint interiors, then the dihedral angles of the $\delta_{i}$ define a critical point of $\mathcal{V}$. 
(In [23], Rivin mainly treats the case where $M$ is a ball and all tetrahedra have a common vertex [see especially Lemma 6.12 and Theorem 14.1 there]: however, the general case requires only minor adjustments. A nice treatment can be found in [9]; see also the proof of Lemma 6.2 in [15], where each interior edge $e$ is associated a natural direction $v_{e} \in T W$ so that the holonomy around $e$ is trivial if and only if $\left.d \mathcal{V}\left(v_{e}\right)=0.\right)$

Note that if $\theta_{1}, \theta_{2}, \theta_{3}$ are the dihedral angles at the edges coming into one (and therefore any) vertex of $\Delta_{i}$, then $\mathcal{V}_{0}\left(\theta_{1}, \theta_{2}, \theta_{3}\right):=\sum_{i=1}^{3} \Lambda\left(\theta_{i}\right)$ is the volume of the ideal tetrahedron of $\mathbb{H}^{3}$ with those dihedral angles, by the Lobachevski formula (this tetrahedron is unique up to isometry of $\mathbb{H}^{3}$ ). Thus $\mathcal{V}(\theta)$ can be interpreted as the sum of the volumes of the tetrahedra $\Delta_{i}$.

Fact 6 The function $\mathcal{V}_{0}$ is continuous and convex on $\Theta:=\left\{\left(\theta_{1}, \theta_{2}, \theta_{3}\right) \in \mathbb{R}_{+}^{3} \mid\right.$ $\left.\theta_{1}+\theta_{2}+\theta_{3}=\pi\right\}$, strictly convex on the interior of $\Theta$, and vanishes on $\partial \Theta$. For any $x \in(0, \pi)$ and any $\omega \in \mathbb{R}$ one has

$$
\left.\frac{d}{d t}\right|_{t=0^{+}} \mathcal{V}_{0}(\pi-x-\omega t, x-(1-\omega) t, t)=+\infty
$$

This expresses the fact that if exactly one of the three angles of an ideal tetrahedron $\Delta$ is 0 , increasing that angle to $\varepsilon \ll 1$ yields a volume increase much greater than $\varepsilon$; note that the same statement is false when two angles of $\Delta$ are 0 . For proofs, refer eg to Propositions 6.6-6.7 of [15] (strict concavity follows from an easy discussion of the second derivative computed there).

Fact 6 implies that the volume functional $\mathcal{V}: W \rightarrow \mathbb{R}$ of Theorem 5 is concave and positive, and extends continuously to a concave function on the (compact) closure $\bar{W}$ of $W$. It moreover implies:

Proposition 7 (Rivin [23]) Suppose $W \neq \varnothing$ and let $\theta_{0} \in \bar{W}$ be a point where $\mathcal{V}$ reaches its maximum. Either

- $\theta_{0}$ belongs to $W$, ie $\theta_{0}(E) \subset \mathbb{R}_{+}^{*}$, in which case $\theta_{0}$ is a (necessarily unique) critical point for $\mathcal{V}$ in $W$; or

- there exists a nonempty list of tetrahedra $\Delta_{i_{1}}, \ldots, \Delta_{i_{s}}$ that have an edge $\epsilon$ such that $\theta_{0}(\epsilon)=0$ : then, each $\Delta_{i_{k}}$ also has an edge $\epsilon^{\prime}$ such that $\theta_{0}\left(\epsilon^{\prime}\right)=\pi$. 


\subsection{Rigidity of solid tori}

In this section we prove a rigidity result for hyperbolic polyhedral solid tori with given dihedral angles (and one ideal vertex). The method is a special case of a generalization of Theorem 5 to spun triangulations.

Consider a once-punctured torus $\tau$ with three ideal edges $e, e^{\prime}, e^{\prime \prime}$ running from the puncture to itself: these edges divide $\tau$ into two ideal triangles. Let $\gamma$ be a nonoriented free homotopy class of simple closed curves in $\tau$, and let $n, n^{\prime}, n^{\prime \prime} \in \mathbb{N}$ be the minimal intersection numbers of $\gamma$ with $e, e^{\prime}, e^{\prime \prime}$ respectively. It is well-known that the triple $\left(n, n^{\prime}, n^{\prime \prime}\right)$ determines the class $\gamma$, and that the largest among $n, n^{\prime}, n^{\prime \prime}$ is the sum of the other two terms.

Let $a, b, c \in[0, \pi)$ be such that $a+b+c=\pi$, and consider coprime positive integers $n_{a}, n_{c}$. We aim to construct a punctured solid torus $X$ (namely a solid torus minus one point of its boundary) with the following properties: the punctured torus $\partial X$ has three ideal edges with exterior dihedral angles $a, b, c$, and the meridian of $X$ intersects these three edges minimally in $n_{a}, n_{a}+n_{c}, n_{c}$ points respectively. We write $n_{b}:=n_{a}+n_{c}$.

Proposition 8 A hyperbolic solid torus $X$ as above exists if and only if $a n_{a}+b n_{b}+$ $c n_{c}>2 \pi$. This solid torus is then unique up to isometry.

Remark 9 The left member of the inequality is the sum of exterior dihedral angles met by a meridian in $\partial X$ : the inequality can thus be seen as a sort of Gauss-Bonnet condition for the compression disk of the solid torus $X$ (see Futer and Guéritaud [12] for a more general construction). In Section 2, we will check that the same condition is also enough for a certain (nonspun) ideal triangulation of $X$ to have angle structures (with respect to $a, b, c$ ), and indeed to be geometrically realized.

Proof If $X$ exists, we can consider its universal cover $U$ which is a complete hyperbolic manifold with locally convex boundary and is thus, by a standard argument, naturally embedded in $\mathbb{H}^{3}$. This space $U$ is the convex hull of the orbit of an ideal point of $\partial_{\infty} \mathbb{H}^{3} \simeq \mathbb{S}^{2}$ under a certain loxodromic $\varphi$ (corresponding to the core curve of $X$ ). We can cone all faces of $U$ to the attracting fixed point of $\varphi$ : this yields a $\varphi$-invariant decomposition of $U$ (minus the axis of $\varphi$ ) into tetrahedra, hence, quotienting out by $\varphi$, a decomposition of the solid torus $X$ (minus the core axis) into two ideal tetrahedra $\Delta, \Delta^{\prime}$. Note that this decomposition has only one interior edge $L$, originating at the puncture of $\partial X$ and spinning towards the core of $X$. Thus, constructing $X$ in general amounts to finding positive dihedral angles for $\Delta, \Delta^{\prime}$ such that: 
(i) The holonomy around $L$ is the identity of $\mathbb{H}^{3}$, ie the six angles around $L$ sum to $2 \pi$ and the six associated tetrahedron shape parameters in $\mathbb{C} \backslash\{0,1\}$ have product equal to 1 . (a definition of holonomy was sketched when we described gluings in Section 1.1 above; for a more precise one, refer eg to Definition 6.3 of [15].)

(ii) The boundary of $\Delta \cup \Delta^{\prime}$ has interior dihedral angles $\pi-a, \pi-b, \pi-c$.

(iii) The holonomy around the core curve of $X$ is also the identity of $\mathbb{H}^{3}$.

Condition (i) above is automatically satisfied because each dihedral angle of $\Delta$ and $\Delta^{\prime}$ is incident to $L$ exactly once. To study Condition (ii), let us fix some notation: let $A B C$ and $A C D$ be two counterclockwise oriented triangles in $\mathbb{C} \subset \mathbb{P}^{1} \mathbb{C} \simeq \partial_{\infty} \mathbb{H}^{3}$; we identify $\triangle$ with the tetrahedron $\infty A B C$ and $\Delta^{\prime}$ with $\infty A C D$, gluing the ideal triangles $\infty A B$ and $\infty D C$ (resp. $\infty A D$ and $\infty B C$ ) together. The interior angles at $A, B, C$ of $\Delta$ are denoted $\delta_{a}, \delta_{b}, \delta_{c}$ respectively. The interior angles at $A, C, D$ of $\Delta^{\prime}$ are denoted $\delta_{c}^{\prime}, \delta_{a}^{\prime}, \delta_{b}^{\prime}$ respectively (see Figure 1).

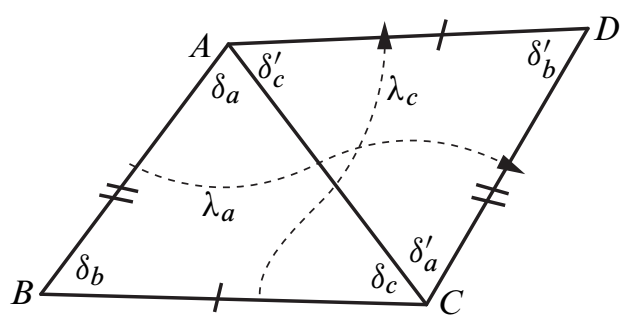

Figure 1: The cusp shapes of $\Delta$ and $\Delta^{\prime}$

Condition (ii) can then be written

$$
\delta_{a}+\delta_{a}^{\prime}=\pi-a, \quad \delta_{b}+\delta_{b}^{\prime}=\pi-b, \quad \delta_{c}+\delta_{c}^{\prime}=\pi-c .
$$

(Indeed, the triangles in Figure 1 represent a triangulation of the punctured torus $\partial\left(\Delta \cup \Delta^{\prime}\right)$, and each interior dihedral angle there is the sum of one angle in $\Delta$ and one angle in $\Delta^{\prime}$.) This implies

$$
\left\{\begin{array}{l}
\left(\delta_{a}, \delta_{b}, \delta_{c}\right)=\left(\frac{\pi-a}{2}+\alpha, \frac{\pi-b}{2}+\beta, \frac{\pi-c}{2}+\gamma\right) \\
\left(\delta_{a}^{\prime}, \delta_{b}^{\prime}, \delta_{c}^{\prime}\right)=\left(\frac{\pi-a}{2}-\alpha, \frac{\pi-b}{2}-\beta, \frac{\pi-c}{2}-\gamma\right)
\end{array}\right.
$$

where

$$
|\alpha|<\frac{\pi-a}{2}, \quad|\beta|<\frac{\pi-b}{2}, \quad|\gamma|<\frac{\pi-c}{2} \quad \text { and } \quad \alpha+\beta+\gamma=0 .
$$


The space of solutions $(\alpha, \beta, \gamma)$ to (2) is the interior of a centrally symmetric affine hexagon $P$ whose edges are given by

$$
\alpha=\frac{\pi-a}{2}, \quad \beta=-\frac{\pi-b}{2}, \quad \gamma=\frac{\pi-c}{2}, \quad \alpha=-\frac{\pi-a}{2}, \quad \beta=\frac{\pi-b}{2}, \quad \gamma=-\frac{\pi-c}{2}
$$

in that order. (It is easy to check that these edges are all nonempty segments if $a, b, c>0$, and that eg the first and fourth edges are reduced to points if and only if $a=0$.) See Figure 2.

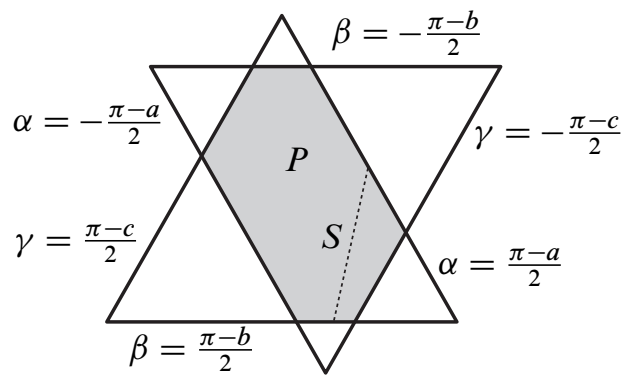

Figure 2: The hexagon $P$ of solutions $(\alpha, \beta, \gamma)$ to (2) and the segment $S$ of spun angle structures. At most one pair of opposite sides of $P$ can be reduced to points, because $a, b, c<\pi$.

Condition (iii) has two components: first, an angular component (linear in terms of the dihedral angles of $\left.\Delta, \Delta^{\prime}\right)$ which will narrow down the space of solutions $(\alpha, \beta, \gamma)$ to the intersection of the interior of $P$ with a certain line. This intersection will be nonempty (namely, an open segment $S$ ) exactly when the inequality of Proposition 8 is satisfied. Second, a scaling component which we will solve by seeking a critical point of a volume functional on $S$.

Angular component Following the notation above (and Figure 1), we refer to the three exterior edges of $X$ as $A B, B C, C A$ : the corresponding exterior dihedral angles are $c, a, b$ respectively. The angular holonomy map is a group homomorphism $h: H_{1}(\partial X, \mathbb{Z}) \rightarrow \mathbb{R}$. Let the oriented closed curve $\lambda_{a}$ (resp. $\lambda_{c}$ ) be a boundary component of a regular neighborhood of the oriented edge $\overrightarrow{B C}$ (resp. $\overrightarrow{B A}$ ), as in Figure 1 . By the conventions (1) above, $h\left(\left[\lambda_{a}\right]\right)=\delta_{a}-\delta_{a}^{\prime}=2 \alpha$ and $h\left(\left[\lambda_{c}\right]\right)=-\delta_{c}+\delta_{c}^{\prime}=-2 \gamma$. The meridian $\mu$ of $X$ is homotopic to $n_{c}\left[\lambda_{a}\right]+n_{a}\left[\lambda_{c}\right]$ (where $n_{a}, n_{c}>0$ ), because this class intersects $n_{a}$ times the edge $B C, n_{b}=n_{a}+n_{c}$ times the edge $A C$, and $n_{c}$ times the edge $B A$. Hence,

$$
h([\mu])=2\left(n_{c} \alpha-n_{a} \gamma\right) .
$$

Using (3), and considering the appropriate vertex of the space $P$ of angle structures, the largest (resp. smallest) possible value of $h([\mu])$ on the closure of $P$ is therefore 
$2\left(n_{c} \frac{\pi-a}{2}+n_{a} \frac{\pi-c}{2}\right)=a n_{a}+b n_{b}+c n_{c}$ (resp. the negative of that number), where we used $a+b+c=\pi$ and $n_{b}=n_{a}+n_{c}$. We conclude that $h([\mu])=2 \pi$ is satisfiable on the interior of $P$ if and only if $a n_{a}+b n_{b}+c n_{c}>2 \pi$, as wished.

Scaling component The scaling holonomy map is a group homomorphism

$$
\eta: H_{1}(\partial X, \mathbb{Z}) \rightarrow \mathbb{R}_{+}^{*}
$$

The sine formula for triangles yields

$$
\eta\left(\left[\lambda_{a}\right]\right)=\frac{\sin \delta_{b}}{\sin \delta_{c}} \frac{\sin \delta_{c}^{\prime}}{\sin \delta_{b}^{\prime}} \quad \text { and } \quad \eta\left(\left[\lambda_{c}\right]\right)=\frac{\sin \delta_{b}}{\sin \delta_{a}} \frac{\sin \delta_{a}^{\prime}}{\sin \delta_{b}^{\prime}},
$$

hence $\quad \eta([\mu])=\eta\left(\left[\lambda_{a}\right]\right)^{n_{c}} \eta\left(\left[\lambda_{c}\right]\right)^{n_{a}}=\left(\frac{\sin \delta_{b}}{\sin \delta_{b}^{\prime}}\right)^{n_{a}+n_{c}}\left(\frac{\sin \delta_{c}}{\sin \delta_{c}^{\prime}}\right)^{-n_{c}}\left(\frac{\sin \delta_{a}}{\sin \delta_{a}^{\prime}}\right)^{-n_{a}}$.

On the other hand, let $S$ be the open segment defined by the intersection of the interior of $P$ with the condition $h([\mu])=2 \pi$, ie $n_{c} \alpha-n_{a} \gamma=\pi$. The tangent space of $S$ is generated by the vector $(\dot{\alpha}, \dot{\beta}, \dot{\gamma})=\left(n_{a},-n_{a}-n_{c}, n_{c}\right)$. The volume functional is by definition

$$
\begin{aligned}
S & \longrightarrow \mathbb{R}^{+} \\
\mathcal{V}:(\alpha, \beta, \gamma) & \longmapsto \mathcal{V}_{0}\left(\delta_{a}, \delta_{b}, \delta_{c}\right)+\mathcal{V}_{0}\left(\delta_{a}^{\prime}, \delta_{b}^{\prime}, \delta_{c}^{\prime}\right)
\end{aligned}
$$

where the angles $\delta_{a}, \ldots, \delta_{c}^{\prime}$ are given by (1). By Fact $6, \mathcal{V}$ is strictly concave on the segment $S$ and achieves its maximum in $S$ (indeed, the endpoints of $S$ belong to the perimeter of the hexagon $P$, but at any point of $\partial P$, at least one of the tetrahedra $\Delta, \Delta^{\prime}$ has exactly one angle whose value is 0 : therefore, $\mathcal{V}$ has unbounded derivative near each endpoint of $S$ ). As a result, $\mathcal{V}$ has a unique (critical) maximum in the open segment $S$.

At that critical point, since $(\dot{\alpha}, \dot{\beta}, \dot{\gamma})=\left(n_{a},-n_{a}-n_{c}, n_{c}\right)$, we have

$$
\begin{aligned}
0=d \mathcal{V}(\dot{\alpha}, \dot{\beta}, \dot{\gamma})= & \dot{\alpha} \Lambda^{\prime}\left(\delta_{a}\right)+\dot{\beta} \Lambda^{\prime}\left(\delta_{b}\right)+\dot{\gamma} \Lambda^{\prime}\left(\delta_{c}\right)-\dot{\alpha} \Lambda^{\prime}\left(\delta_{a}^{\prime}\right)-\dot{\beta} \Lambda^{\prime}\left(\delta_{b}^{\prime}\right)-\dot{\gamma} \Lambda^{\prime}\left(\delta_{c}^{\prime}\right) \\
= & -\dot{\alpha} \log \left|2 \sin \delta_{a}\right|-\dot{\beta} \log \left|2 \sin \delta_{b}\right|-\dot{\gamma} \log \left|2 \sin \delta_{c}\right| \\
& +\dot{\alpha} \log \left|2 \sin \delta_{a}^{\prime}\right|+\dot{\beta} \log \left|2 \sin \delta_{b}^{\prime}\right|+\dot{\gamma} \log \left|2 \sin \delta_{c}^{\prime}\right| \\
= & \log \left(\left(\frac{\sin \delta_{a}}{\sin \delta_{a}^{\prime}}\right)^{-n_{a}}\left(\frac{\sin \delta_{b}}{\sin \delta_{b}^{\prime}}\right)^{n_{a}+n_{c}}\left(\frac{\sin \delta_{c}}{\sin \delta_{c}^{\prime}}\right)^{-n_{c}}\right)=\log \eta([\mu]) .
\end{aligned}
$$

At the critical point of $\mathcal{V}$ in $S$, we therefore have the following values for the holonomy maps: $h([\mu])=2 \pi$ (rotational component) and $\eta([\mu])=1$ (scaling component). This precisely means that the metric completion of $\Delta \cup \Delta^{\prime}$ is the solid torus $X$ endowed with a spun triangulation of two tetrahedra whose tips spin around the core curve. Moreover, any realization of $X$ with the prescribed dihedral angles yields a spun triangulation 
into positively oriented tetrahedra $\left(\Delta, \Delta^{\prime}\right)$, because we can always cone the faces of the (convex) universal cover $U \subset \mathbb{H}^{3}$ of $X=U / \varphi$ to the attracting fixed point of $\varphi$. Since the critical point of $\mathcal{V}$ in $S$ is unique, we have therefore proved that $X$ itself is unique up to isometry.

\section{Farey combinatorics in solid tori}

Let $X$ be a compact solid torus, minus one point of its boundary; call this removed point the puncture.

In this section we will first describe a certain combinatorial decomposition $\mathcal{D}$ of $X$ into ideal tetrahedra, relative to a given ideal triangulation of $\partial X$ (into two ideal triangles). This is essentially similar to a construction for closed manifolds that appears in the function standard_torus_form() in [27, close_cusps.c]. This layering construction is also analyzed in great detail by Jaco and Rubinstein [17]. We next go on to find a geometric realization of $\mathcal{D}$, using the ideas of Section 1.

\subsection{The Farey graph}

Identify the boundary at infinity of the hyperbolic plane $\mathbb{H}^{2}$ to the circle $\mathbb{P}^{1} \mathbb{R}$, endowed with the action of $\mathrm{PSL}_{2}(\mathbb{Z})$. We assume that $0,1, \infty$ lie counterclockwise in that order on $\partial_{\infty} \mathbb{H}^{2} \simeq \mathbb{P}^{1} \mathbb{R}$. Consider the subset $\mathbb{P}^{1} \mathbb{Q}$ of $\mathbb{P}^{1} \mathbb{R}$. We measure the "proximity" of two elements $q=y / x$ and $q^{\prime}=y^{\prime} / x^{\prime}$ of $\mathbb{P}^{1} \mathbb{Q}$ (given as ratios of coprime integers) by computing their wedge

$$
q \wedge q^{\prime}:=\left\|\begin{array}{ll}
y & y^{\prime} \\
x & x^{\prime}
\end{array}\right\| \in \mathbb{N} \quad \text { (absolute value of the determinant). }
$$

If we draw a straight line in $\mathbb{H}^{2}$ from $q$ to $q^{\prime}$ each time $q \wedge q^{\prime}=1$, we obtain the Farey triangulation of $\mathbb{H}^{2}$. Alternatively, this triangulation can be defined by reflecting the ideal triangle $1 \infty 0$ in its sides ad infinitum.

Fix an identification (homeomorphism) between the punctured torus $\partial X$ and $\mathbb{T}:=$ $\left(\mathbb{R}^{2} \backslash \mathbb{Z}^{2}\right) / \mathbb{Z}^{2}$. We assume that the canonical orientation of $\mathbb{T}$ (induced by $\mathbb{R}^{2}$ ), followed by the outward-pointing normal of $\partial X$, coincides with the positive orientation on $X$. The segment from $(0,0)$ to $(x, y)$ in $\mathbb{R}^{2}$ (where $x, y$ are coprime integers) projects to a properly embedded (open) arc $\gamma$ in $\partial X$ : we say that $y / x \in \mathbb{P}^{1} \mathbb{Q}$ is the slope of $\gamma$. An edge $E$ of the Farey triangulation (or: a Farey edge) corresponds to a pair of disjoint arcs $\gamma, \gamma^{\prime}$ in $\partial X$; the slopes of $\gamma, \gamma^{\prime}$ are the two ends of $E$ in $\mathbb{P}^{1} \mathbb{Q}$ and the complement of $\gamma \cup \gamma^{\prime}$ in $\partial X$ is an ideal quadrilateral. Similarly, Farey triangles 
(such as $1 \infty 0$ ), having three vertices in $\mathbb{P}^{1} \mathbb{Q}$, correspond to triples of disjoint arcs $\gamma, \gamma^{\prime}, \gamma^{\prime \prime}$ in $\partial X$ which define a decomposition of $\partial X$ into two ideal triangles. Finally, note that we can also associate a slope in $\mathbb{P}^{1} \mathbb{Q}$ to the meridional closed curve $\mu$ of the solid torus $X$ : namely, the slope of the unique properly embedded arc $\mu^{\prime}$ which (possibly after isotopy) does not intersect $\mu$.

Let $p q r$ be a Farey triangle, and suppose $m \in \mathbb{P}^{1} \mathbb{Q} \backslash\{p, q, r\}$ is the slope of the meridian of $X$. By convention, we will suppose that the Farey edge $p q$ separates $r$ from $m$, and that $p q m$ is not a Farey triangle (so $m$ is "far enough" from the triangle $p q r$ ). Endow the punctured torus $\partial X$ with the ideal triangulation associated to $p q r$ (which we call the $p q r$-triangulation). In Section 2.2, we will be preoccupied with decomposing $X$ into ideal tetrahedra with faces (ideal triangles) glued in pairs, in such a way that exactly two ideal triangles remain free, and give the $p q r$-triangulation of $\partial X$.

\subsection{An ideal triangulation of the solid torus}

The idea is to follow a path $\ell$ in the Farey triangulation, transverse to the Farey edges, from the ideal vertex $r$ to the ideal vertex $m$. We assume that the path $\ell$ crosses each Farey triangle at most once, ie never backtracks. The sequence of Farey triangles that $\ell$ encounters is then completely determined (so we can take $\ell$ to be eg a geodesic ray): these triangles are

$$
\left(T_{0}, T_{1}, \ldots, T_{N}\right)=\left(p q r, p q r^{\prime}, \ldots, m s t\right)
$$

where $s, t$ belong to $\mathbb{P}^{1} \mathbb{Q}$ and the symmetry of axis $p q$ takes $r$ to $r^{\prime}$. Note that by assumption, $N \geq 2$. See Figure 5 .

For each $0<i<N$, we can then consider a properly embedded punctured torus $\tau_{i} \subset X$ isotopic to $\partial X$ (properness here means that by intersecting $\tau_{i}$ with a basis of neighborhoods of the puncture of $X$, we get a basis of neighborhoods of the puncture of $\tau_{i}$ ). We can assume that the $\tau_{i}$ are disjoint and that $\tau_{i}$ separates $\partial X$ from $\tau_{i+1}$ (ie $\tau_{i+1}$ lies in the solid torus $X$ "inward" from $\tau_{i}$ ). Endow $\tau_{i}$ with the triangulation associated to the Farey triangle $T_{i}$ - for that purpose we also count $\partial X$ as $\tau_{0}$. Note that two consecutive punctured tori $\tau_{i-1}, \tau_{i}$ always have two edge slopes in common (these slopes are the ends of the Farey edge $T_{i-1} \cap T_{i}$ ). Thus, we can isotope $\tau_{1}$ until its edges of slopes $p, q$ coincide with those of $\tau_{0}=\partial X$; then isotope $\tau_{2}$ until two of its edges coincide with the edges of similar slopes in $\tau_{1}$; then isotope $\tau_{3}$ until it intersects $\tau_{2}$ along two edges, etc.

At the end of this process, the space comprised between $\tau_{i-1}$ and $\tau_{i}$, for each $0<$ $i<N$, is a (combinatorial) ideal tetrahedron $\Delta_{i}$ with four of its edges identified in 
opposite pairs. These tetrahedra $\Delta_{i}$, with the combinatorial gluing that arises from the construction above, are by definition those of our decomposition $\mathcal{D}$ of $X$. (Since $N \geq 2$, there is at least one tetrahedron $\Delta_{i}$. Our "half-shift" convention $\partial \Delta_{i}=\tau_{i-1} \cup \tau_{i}$, or equivalently $\tau_{i}=\Delta_{i} \cap \Delta_{i+1}$, is arbitrary). In order to homotopically "kill" the meridian of the solid torus $X$, it only remains to describe the gluing of the last surface $\tau_{N-1}$ to itself.

If $T_{N}=m s t$ is the last Farey triangle, let $T_{N-1}=m^{\prime} s t$ be the next-to-last, associated to the surface $\tau_{N-1}$. We fold $\tau_{N-1}$ along its edge of slope $m^{\prime}$, gluing the two adjacent faces (ideal triangles) $F^{\prime}, F^{\prime \prime}$ to one another to obtain a single ideal triangle $F$. Intrinsically, $F$ is an ideal Möbius band, ie a compact Möbius band minus one point of its boundary. Indeed, from an (ideal) triangle $A B C$, one can construct an (ideal) Möbius band $F$ with boundary $A C$, by gluing the oriented edge $A B$ to $B C$ : the (punctured) torus $\tau_{N-1}=F^{\prime} \cup F^{\prime \prime}$ then just wraps around this (ideal) Möbius band $F$, like the boundary of a regular neighborhood of an embedding of $F$ in $\mathbb{R}^{3}$. See Figure 3 .
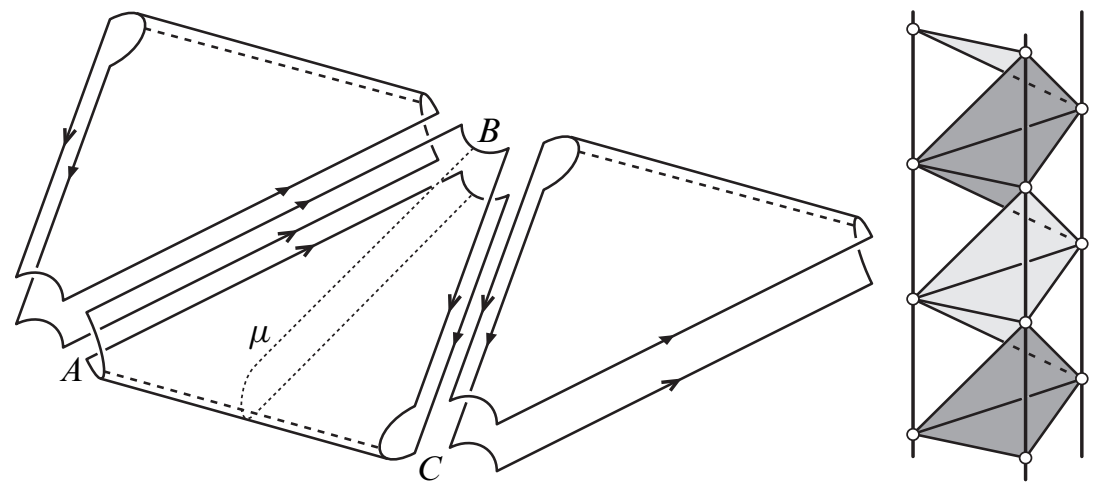

Figure 3: Left: A punctured torus (shown are 3 folded copies of a fundamental domain; arrows are identified) wraps around an ideal Möbius band. The meridian arc $\mu$, of slope $m$, becomes homotopically trivial. The dotted folding edge $A C$ has slope $m^{\prime}$. Right: Part of the universal cover of the same Möbius band (shaded) and the tetrahedron $\Delta_{N-1}$ glued to it.

\subsection{Angle structures}

We proceed to describe positive angle structures for the tetrahedra $\Delta_{i}$, where $1 \leq i \leq$ $N-1$ (the argument is reminiscent of [15] and [16], although the solution space will look quite different). More precisely, consider reals

$$
\theta_{p}, \theta_{q}, \theta_{r} \text { such that }\left\{\begin{aligned}
\theta_{p}+\theta_{q}+\theta_{r} & =\pi ; \\
\theta_{p}, \theta_{q} & \geq 0 ; \\
\pi>\theta_{r} & >0 .
\end{aligned}\right.
$$


We will look for angle structures on the $\Delta_{i}$ such that the interior dihedral angles of $X$ at the edges of slope $p, q, r$ in $\partial X$ are $\pi-\theta_{p}, \pi-\theta_{q}, \pi-\theta_{r}$ respectively. Note that we do not allow $\theta_{r}$ to vanish: indeed, $\pi-\theta_{r}$ will be a dihedral angle of the first tetrahedron $\Delta_{1}$. (If the solid torus $X$ admits a geometric realization in which $\theta_{r}=0$, we can always remove this flat tetrahedron $\Delta_{1}$ and see $\partial X$ as being endowed with the $p q r^{\prime}$-triangulation, where $r^{\prime} \in \mathbb{P}^{1} \mathbb{Q}$ is the symmetric image of $r$ with respect to the Farey edge $p q$.

Proposition 10 An angle structure satisfying (5), also called a $\left(\theta_{p}, \theta_{q}, \theta_{r}\right)$-angle structure, exists if and only if $(m \wedge p) \theta_{p}+(m \wedge q) \theta_{q}+(m \wedge r) \theta_{r}>2 \pi$.

Remark 11 It is easy to check that $m \wedge r=(m \wedge p)+(m \wedge q)-$ eg by reducing to the case $(p, q)=(0, \infty)$ and using the $\operatorname{PSL}_{2}(\mathbb{Z})$-invariance of the $\wedge$ notation. Thus, by (5), the inequality of Proposition 10 is automatically true unless $\min \{m \wedge p, m \wedge q\}=1$. For example, if $(m \wedge p, m \wedge q)=(1,1)$, the condition is always false (recall we required that $p q m$ not be a Farey triangle); if $(m \wedge p, m \wedge q)=(2,1)$, it amounts to $\theta_{r}>\theta_{q}$. The equilateral triangle in Figure 4 shows the full parameter space for the triple $\left(\theta_{p}, \theta_{q}, \theta_{r}\right)$ : shades indicate how many slopes $m$ fail to satisfy the condition of Proposition 10, where we allow $m$ to range over all of $\mathbb{P}^{1} \mathbb{Q}$ rather than just over the arc $\widehat{p q}$ (when $m$ belongs to one of the arcs $\overparen{q r}, \overparen{r p}$, we construct the same ideal triangulations, up to a permutation of $p, q, r)$.
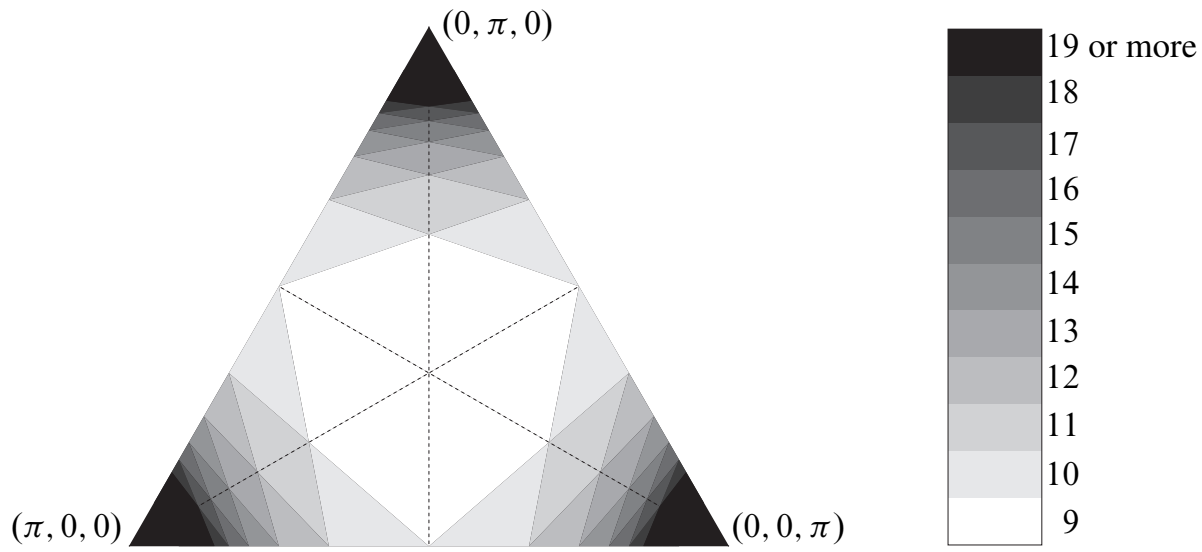

Figure 4: Parameter space for the triple $\left(\theta_{p}, \theta_{q}, \theta_{r}\right)$ and numbers of "forbidden" slopes $m$ (the brighter, the fewer)

Proof of Proposition 10 The tetrahedra $\Delta_{i}$ are naturally associated to the Farey edges $e_{i}=T_{i-1} \cap T_{i}$ that the path $\ell$ crosses. Orient $\ell$ from $T_{0}$ to $T_{N}$. If $e_{i}$ and $e_{i+1}$ 
share their Right (resp. Left) end with respect to the orientation of $\ell$, we say that $\ell$ makes a Right (resp. a Left) between $e_{i}$ and $e_{i+1}$ (or: at $T_{i}$ ). Thus, $\ell$ defines a word $\Omega=R L L \ldots R$ of length $N-1$ in the letters $R, L:$ for each $i \in\{1,2, \ldots, N-1\}$ there is a tetrahedron $\Delta_{i}$ and a letter $\Omega_{i} \in\{R, L\}$. If $(p, q, r)=(0, \infty,-1)$, then the lengths of the syllables $R^{n}$ and $L^{n}$ of $\Omega$ are exactly the integers in the continued fraction expansion of the rational $m$, as referred to in Theorem 1 .

Note that no letter $R$ or $L$ is associated to the very first Farey triangle $T_{0}=p q r$, because the line $\ell$ does not "enter" $T_{0}$ through $p r$ rather than through $q r$. We nevertheless decide to place an extra letter $\Omega_{0} \in\{R, L\}$ in front of the word $\Omega$, so that $\Omega$ becomes of length $N$ and starts with either $R R$ or $L L$. This convention is totally artificial (the other choice would be equally good), but making a choice here will allow us to streamline the notation in our argument. Up to switching $p$ and $q$, we can now assume that $\ell$ enters the Farey triangle $T_{0}$ through the edge $p r$, and leaves through $p q$. See Figure 5.

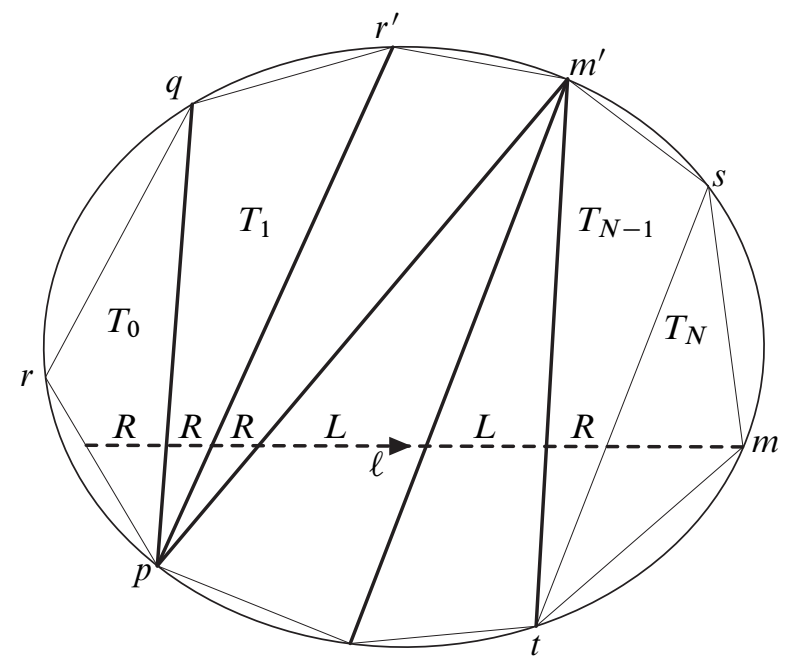

Figure 5: The Farey graph. The 5 thick lines $T_{i-1} \cap T_{i}$ (where $1 \leq i \leq 5$ ) correspond to the tetrahedra $\Delta_{i}$.

Definition 12 If $\Omega_{i-1} \neq \Omega_{i}$, we say that $\Delta_{i}$ is a hinge tetrahedron. Otherwise, we call $\Delta_{i}$ nonhinge. For example, following our convention, $\Delta_{1}$ is nonhinge.

To compute angle structures, it will be useful to describe the cusp triangulation associated to the ideal triangulation $\left\{\Delta_{i}\right\}_{1 \leq i \leq N-1}$ of $X$. Since each pleated punctured torus $\tau_{i}$ has one ideal vertex and three edges, each with two ends, the link of the ideal 
vertex of $\tau_{i}$ is a hexagon $H_{i}$ (the pleating angles of $\tau_{i}$ are the exterior angles of $H_{i}$ ). We are going to define the dihedral angles of the ideal tetrahedra $\Delta_{i}$ in terms of the pleating angles of the $\tau_{i}$. Note that the hexagon $H_{i}$ has a central symmetry induced by the hyperelliptic involution of the punctured torus $\tau_{i}$ (rotation of $180^{\circ}$ around the puncture, which exchanges the ends of each edge of $\tau_{i}$ ).

Let $\xi \eta \zeta=T_{i-1}$ and $\xi \eta \zeta^{\prime}=T_{i}$ be two consecutive Farey triangles, so that the Farey vertex $\xi$ (resp. $\eta$ ) lies to the right (resp. left) of the oriented axis $\ell$. The tetrahedron $\Delta_{i}$ has

- two opposite edges carrying the same dihedral angle $x_{i}$ and identified to just one edge, of slope $\xi$, in the triangulation of the solid torus (for the time being, $x_{i}$ is just a formal variable);

- two opposite edges carrying the same dihedral angle $y_{i}$ and identified to just one edge, of slope $\eta$, in the triangulation (similarly, $y_{i}$ is formal);

- two opposite edges which carry the same (formal) dihedral angle $z_{i}$, and which coincide with the edges of slope $\zeta$ and $\zeta^{\prime}$ in the triangulation.

As in any angle structure, the relationship $x_{i}+y_{i}+z_{i}=\pi$ must hold between the formal variables.

The vertices of the hexagon $H_{i-1}$ (resp. $H_{i}$ ) are the links of edges of slopes $\xi, \eta, \zeta$ (resp. $\xi, \eta, \zeta^{\prime}$ ). We can write these labels $\xi, \eta, \zeta, \zeta^{\prime}$ at the vertices of $H_{i-1}$ and $H_{i}$ : see Figure 6 (left).

Observation 13 By construction, the vertex of the hexagon $H_{i-1}$ labelled $\zeta$ has an interior angle of $z_{i}$, while the vertex of the hexagon $H_{i}$ labelled $\zeta^{\prime}$ has an interior angle of $2 \pi-z_{i}$. This comes from the fact that the boundary of the tetrahedron $\Delta_{i}$ is exactly the union of the two pleated punctured tori $\tau_{i-1}$ and $\tau_{i}$ (with vertex links $\left.H_{i-1}, H_{i}\right)$.

As a consequence, we can determine the three interior angles of the hexagon $H_{i}$ (each angle occurs twice, by central symmetry of $H_{i}$ ):

$$
2 \pi-z_{i}, \quad z_{i+1}, \quad z_{i}-z_{i+1} .
$$

Indeed, the first two of these numbers are given by Observation 13 (shifting indices by one for $z_{i+1}$ ); the third is given by the property that the six angles of $H_{i}$ should add up to $4 \pi$. See Figure 6, (right).

We can in turn write the numbers (6) in the corners of the Farey triangle $T_{i}$ : namely, $2 \pi-z_{i}$ is in the corner opposite the Farey edge $T_{i-1} \cap T_{i}$; similarly $z_{i+1}$ is in the 

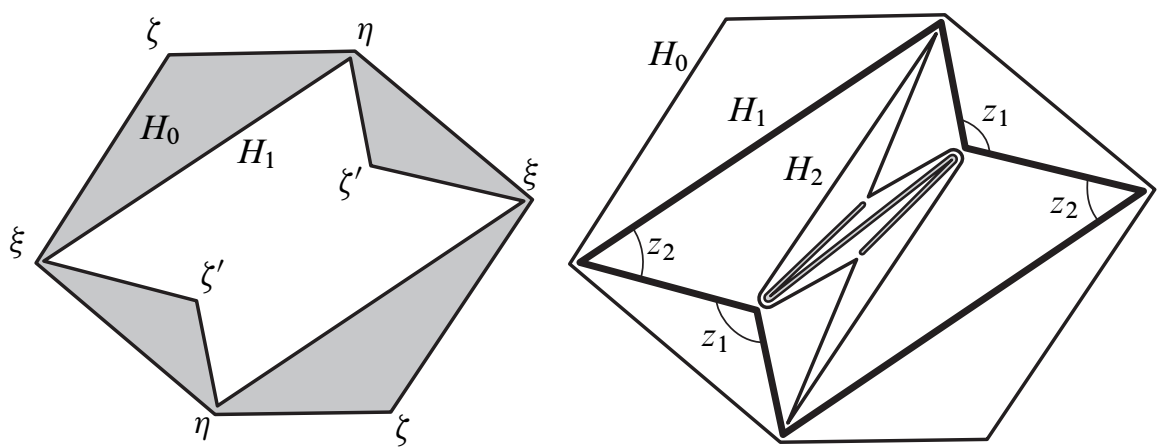

Figure 6: Left: Two consecutive hexagons $H_{0}, H_{1}$ in the cusp link, with vertices labelled by elements of $\mathbb{P}^{1} \mathbb{Q}$. The four (similar) grey triangles are the vertex links of the ideal tetrahedron $\Delta_{1}$. Right: The full sequence of hexagons $H_{0}, \ldots, H_{3}$, where $H_{3}$ is collapsed to a broken line of 3 segments. The angles $z_{1}$ and $z_{2}$ of the tetrahedra $\Delta_{1}$ and $\Delta_{2}$ are marked; together they determine the interior angles (6) of $H_{1}$.

corner opposite the Farey edge $T_{i} \cap T_{i+1}$; and $z_{i}-z_{i+1}$ is in the third corner, at the Farey vertex $T_{i-1} \cap T_{i+1}$. See Figure 7 .

The above operation can be performed for all indices $i \in\{1, \ldots, N-2\}$. For $i=N-1$, there is no tetrahedron " $\Delta_{N}$ "; hence, a priori, no parameter $z_{N}$. However, if $m^{\prime} \in \mathbb{P}^{1} \mathbb{Q}$ is the vertex of the Farey triangle $T_{N-1}$ opposite the Farey edge $T_{N-1} \cap T_{N}$ in $T_{N-1}$, then the interior angle of the (collapsed) hexagon $H_{N-1}$ at the vertex labelled $m^{\prime}$ is precisely 0 , by definition of our folding of the pleated surface $\tau_{N-1}$ onto itself. This folding thus corresponds to asking that

$$
z_{N}=0 .
$$

Under this convention, the other angles of the collapsed hexagon $H_{N-1}$ are then given by the same formulas (6), with $i=N-1$.

Finally, we perform an analogous construction at $i=0$ (our assumptions imply that $H_{0}$ is convex, with angles $\pi-\theta_{p}, \pi-\theta_{q}, \pi-\theta_{r}$ ). There is no tetrahedron " $\Delta_{0}$ "; hence, a priori, no parameter $z_{0}$. However, the interior angle of $H_{0}$ at the vertex labelled $r$ is $\pi-\theta_{r}$, which entails $z_{1}=\pi-\theta_{r}$. Similarly, the interior angle of $H_{0}$ at the vertex labelled $q$ is $\pi-\theta_{q}$, which entails $z_{0}=2 \pi-\left(\pi-\theta_{q}\right)=\pi+\theta_{q}$. To summarize:

\section{Proposition 14 Under the full set of assumptions}

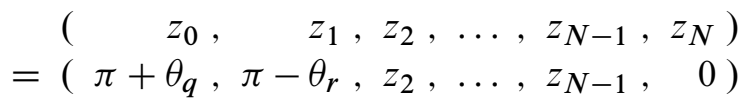


(where the values of $\left(z_{2}, \ldots, z_{N-1}\right)$ remain to be chosen), the angles of the hexagons $\left\{H_{i}\right\}_{0 \leq i \leq N-1}$ given by (6) define all the $\left(\theta_{p}, \theta_{q}, \theta_{r}\right)$-angle structures.

To get angle structures, we must only choose the $z_{2}, \ldots, z_{N-1}$ in the interval $(0, \pi)$ so that all dihedral angles of $\Delta_{i}$ are positive for $1 \leq i \leq N-1$, which we do now.

Denote by $\xi$ (resp. $\eta$ ) the right (resp. left) end of the Farey edge $T_{i-1} \cap T_{i}$. By construction, $x_{i}$ (resp. $y_{i}$ ) is half the difference between the angles of hexagons $H_{i-1}$ and $H_{i}$ at the vertex labelled $\xi$ (resp. $\eta$ ) in the cusp link, ie half the difference between the numbers written in the $\xi$-corner (resp. the $\eta$-corner) of the Farey triangles $T_{i-1}$ and $T_{i}$ in the Farey diagram. (The factor one-half comes from the identification of pairs of opposite edges in the ideal tetrahedron $\Delta_{i}$.) In Figure 7 we show what these numbers are, according to whether the line $\ell$ makes Rights or Lefts at the Farey triangles $T_{i-1}$ and $T_{i}$ : we use only (6) and the shorthand

$$
(a, b, c):=\left(z_{i-1}, z_{i}, z_{i+1}\right) .
$$
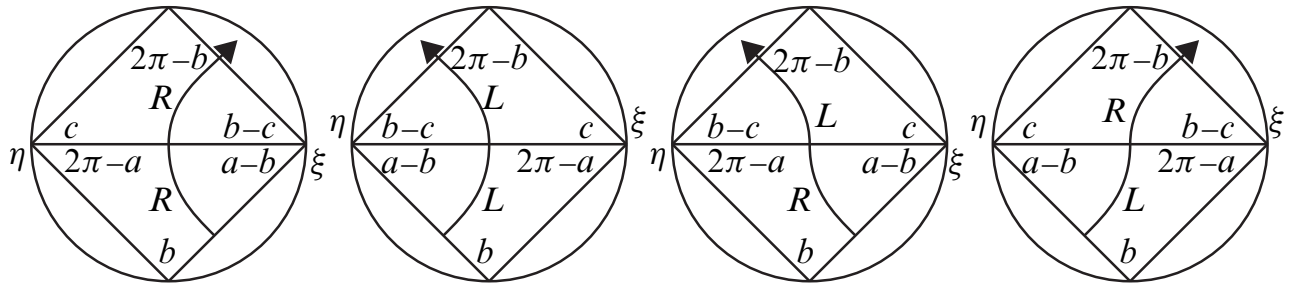

Figure 7: The Farey triangles $T_{i-1}$ (lower) and $T_{i}$ (upper), with corner labels

It follows that the values of $x_{i}$ and $y_{i}$ in terms of the $z_{i}$ are given by Table (9) - in the first line of the table, we recall the nature of the tetrahedron (or cell) $\Delta_{i}$, and the natural positions of $a, b, c$, interspersed with the letters of the word $\Omega$.

\begin{tabular}{|c|c|c|c|c|}
\hline 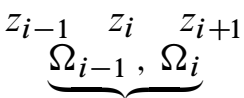 & $\underbrace{a{ }^{R, R}{ }^{c}}$ & ${ }^{a} \underbrace{L, b{ }^{c}{ }^{c}}$ & $\underbrace{a{ }^{R, b}, L}{ }^{c}$ & $\underbrace{a} \underbrace{b, R^{c}}$ \\
\hline Cell $\Delta_{i}$ is... & Nonhinge & Nonhinge & Hinge & Hinge \\
\hline \multirow{2}{*}{$x_{i}$} & $a-2 b+c$ & $\pi-a+c$ & $a-b-c$ & $a-a+b-c$ \\
\hline & 2 & 2 & 2 & 2 \\
\hline \multirow[b]{2}{*}{$y_{i}$} & $a+c$ & $a-2 b+c$ & $a+b-c$ & $a-b-c$ \\
\hline & & 2 & 2 & 2 \\
\hline$z_{i}$ & $b$ & $b$ & $b$ & $b$ \\
\hline
\end{tabular}


From Table (9), we can read off the condition for all $x_{i}$ and $y_{i}$ and $z_{i}$ to be positive. Still using the notation $(a, b, c)=\left(z_{i-1}, z_{i}, z_{i+1}\right)$ (and recalling that the values of $z_{0}, z_{1}$ are forced by (7)), these conditions are

$$
\left\{\begin{array}{lll}
a>b+c & \text { if } \Delta_{i} \text { is a hinge cell } & \text { (hinge condition); } \\
a+c>2 b & \text { if } \Delta_{i} \text { is not a hinge } & \text { (convexity condition); } \\
0<z_{i}<\pi & \text { for all } 2 \leq i \leq N-1 & \text { (range condition); } \\
z_{2}<\pi-\theta_{q} & \text { (follows from the case } i=1, \text { a nonhinge index). }
\end{array}\right.
$$

The last condition is needed for $\pi-\left(z_{0}+z_{2}\right) / 2$ (namely, $x_{1}$ or $\left.y_{1}\right)$ to be positive, because $z_{0}=\pi+\theta_{q}$ (unlike other $z_{i}$ ) is larger than $\pi$. Note that by (7), the convexity condition at $i=1$ also implies $z_{2}>\pi-\theta_{q}-2 \theta_{r}$. This is compatible with the last condition of (10) since $\theta_{r}>0$ by (5).

To actually find $\left(z_{2}, \ldots, z_{N-1}\right)$ satisfying (10), thus proving Proposition 10, we now distinguish two cases.

Case 1 (None of the $\Delta_{i}$ are hinge cells.) In this case, we are reduced to finding a sequence of the form (7) that is convex, decreasing, and satisfies $z_{2}<\pi-\theta_{q}$. This is clearly possible if and only if

$$
\begin{aligned}
\left(\pi+\theta_{q}\right)-N\left(\theta_{r}+\theta_{q}\right) & <0, \\
(N-1) \theta_{q}+N \theta_{r} & >\pi, \\
\theta_{p}+N \theta_{q}+(N+1) \theta_{r} & >2 \pi,
\end{aligned}
$$

ie

where the last line follows from (5). It is easy to check that under the normalization $(p, q)=(\infty, 0)$ and $r \in\{+1,-1\}$ (one of which can be assumed up to applying an element of $\left.\operatorname{PSL}_{2}(\mathbb{Z})\right)$, the slope $m \in \mathbb{P}^{1} \mathbb{Q}$ is, up to sign, the integer $N$ : indeed, all the letters of the word $\Omega$ are equal and the Farey triangle $T_{i}$ has vertices $\infty, i, i-1$ if $r=1$ (and $\infty,-i,-i+1$ if $r=-1$ ). The last line of the computation above thus becomes

$$
(m \wedge p) \theta_{p}+(m \wedge q) \theta_{q}+(m \wedge r) \theta_{r}>2 \pi,
$$

proving Proposition 10 in this case.

Case 2 (Some $\Delta_{i}$ are hinge cells.) By Remark 11, the inequality of Proposition 10 is vacuous in this case. Let us therefore just construct a sequence of the form (7) that satisfies (10). Let $h \in\{2,3, \ldots, N-1\}$ be the smallest hinge index. We can easily choose a strictly convex, positive, decreasing sequence

$$
\begin{aligned}
& \left(z_{0}, \quad z_{1}, z_{2}, \ldots, z_{h-1}, z_{h}\right) \\
& =\left(\pi+\theta_{q}, \pi-\theta_{r}, z_{2}, \ldots, z_{h-1}, z_{h}\right)
\end{aligned}
$$


satisfying $z_{2}<\pi-\theta_{q}$. We construct the rest of the sequence $\left(z_{i}\right)$ backwards, descending from the index $i=N$ down to $i=h$. First set $z_{N}^{\prime}=0$ and $z_{N-1}^{\prime}=1$. For each $i$ such that $N-2 \geq i \geq h+1$, pick (inductively) a value of $z_{i}^{\prime}$ such that $(a, b, c):=\left(z_{i}^{\prime}, z_{i+1}^{\prime}, z_{i+2}^{\prime}\right)$ satisfies the concavity or hinge condition of (10), according to whether $\Delta_{i+1}$ is a hinge cell or not (for example, $z_{i}^{\prime}=3 z_{i+1}^{\prime}$ will always do). The sequence $\left(z_{h+1}^{\prime}, \ldots, z_{N-1}^{\prime}\right)$ is clearly positive and decreasing. We then set

$$
z_{i}:=\varepsilon z_{i}^{\prime} \quad \text { for all } h+1 \leq i \leq N .
$$

It is immediate to check that the hinge condition " $a>b+c$ " of (10) is verified by the triple $(a, b, c)=\left(z_{h-1}, z_{h}, z_{h+1}\right)$ as soon as

$$
0<\varepsilon<\frac{z_{h-1}-z_{h}}{z_{h+1}^{\prime}} .
$$

Thus, by choosing such an $\varepsilon$, we have found a sequence $\left(z_{i}\right)$ of the form (7).

Proposition 10 is proved.

\subsection{Volume maximization}

Denote by (10') the system (10) in which all strong inequalities have been replaced by weak ones, and let $W$ denote the compact polyhedron of solutions $\left(z_{i}\right)$ of the form (7) to the system (10') - so the interior of $W$ is the space of angle structures. The volume functional $\mathcal{V}: W \rightarrow \mathbb{R}^{+}$associates to every point $z$ of $W$ the sum of the volumes of the ideal tetrahedra $\Delta_{i}$ with nonnegative angles $x_{i}, y_{i}, z_{i}$ given by Table (9).

Suppose that $\theta_{p}, \theta_{q}, \theta_{r}$ satisfy (5) and the inequality of Proposition 10 (hence $W \neq \varnothing$ ). We henceforth assume that the point $z=\left(z_{i}\right) \in W$ realizes the maximum of $\mathcal{V}$ over $W$, and we aim to prove:

Proposition 15 The point $z$ is a solution of (10), not just (10') - ie all $\Delta_{i}$ have only positive angles.

Proof Observe that the sequence $\left(z_{0}, \ldots, z_{N}\right)$ is nonnegative and nonincreasing. This follows from (10') by an immediate downward induction (starting at $z_{N}$ ).

By Proposition 7, we know that if $\Delta_{i}$ is flat, ie has a vanishing dihedral angle, then its triple of angles is of the form $(0,0, \pi)$, up to permutation. Thus, by Table (9), $\Delta_{i}$ is flat exactly when $z_{i} \in\{0, \pi\}$. By monotonicity, since $z_{1}=\pi-\theta_{r}<\pi$, the only flat tetrahedra $\Delta_{i}$ actually satisfy $z_{i}=0$. Still by monotonicity, it then follows that $z_{i+1}=0$ as well. Let $i$ be the smallest index such that $z_{i}=0$. An easy discussion, using Table (9), shows that the only possible value of $z_{i-1}$ that implies $\left\{x_{i}, y_{i}\right\}=\{0, \pi\}$ is 
$z_{i-1}=2 \pi$ (recall here the $a-b-c$-Equation (8)). This is impossible: only $z_{0}=\pi+\theta_{q}$ is allowed to be larger than $\pi$, but we have $\theta_{q}<\pi$ by (5).

Corollary 16 The point $z$ defines a complete hyperbolic structure on the punctured solid torus $X=\Delta_{1} \cup \cdots \cup \Delta_{N-1}$, with exterior dihedral angles $\theta_{p}, \theta_{q}, \theta_{r}$ on $\partial X$.

Proof By Theorem 5, this follows from the fact that $z$ is critical for the volume functional $\mathcal{V}: W \rightarrow \mathbb{R}$.

An alternative proof would closely follow that of [15, Lemma 6.2]: to each interior edge $E$ of $X$ is associated a certain line $L_{E}$ in the tangent space $T_{z} W$, such that the vanishing of the derivative of $\mathcal{V}$ along $L_{E}$ expresses the fact that the hyperbolic metric near $E$ is complete.

\section{Handedness}

In this section, we discuss the handednesses of certain elements in the fundamental group of the (complete, hyperbolic) punctured solid torus $X$. These results will be useful in establishing the inequalities leading to Theorem 1 (which is proved in the next section).

Definition 17 For any $g \in \mathrm{GL}_{2}(\mathbb{C})$, define the handedness of $g$ by

$$
\operatorname{hand}(g):=\frac{(\operatorname{Tr} g)^{2}}{\operatorname{Det} g} \text {. }
$$

Note that hand $(g)=\operatorname{hand}\left(g^{-1}\right)=\operatorname{hand}(r g)$ for all $r \in \mathbb{C}^{*}$. Therefore, hand factors through a map $\mathrm{PSL}_{2}(\mathbb{C}) \rightarrow \mathbb{C}$, also noted hand. Call a loxodromy of $\mathbb{H}^{3}$ left-handed (resp. right-handed) when it is conjugate to $z \mapsto \alpha z$ with $|\alpha|>1$ and $\operatorname{Im}(\alpha)>0$ (resp. $|\alpha|>1$ and $\operatorname{Im}(\alpha)<0)$. Left-handed loxodromies are "corkscrew" motions, the motion of a dancer who jumps upwards while spinning to her left. It is easy to check that the Möbius transformation associated to $g$ is left- (resp. right-) handed if and only if $\operatorname{Im}($ hand $(g))$ is positive (resp. negative).

Let $U$ be the universal cover of the solid torus $X=\bigcup_{i=1}^{N-1} \Delta_{i}$. Since $U$ is a complete hyperbolic manifold with locally convex boundary, the developing map $U \rightarrow \mathbb{H}^{3}$ is an embedding. Thus $U \subset \mathbb{H}^{3}$ is the convex hull in $\mathbb{H}^{3}$ of the orbit of an ideal point $v$ under a certain loxodromy

$$
\varphi \in \operatorname{Isom}^{+}\left(\mathbb{H}^{3}\right) \simeq \mathrm{PSL}_{2}(\mathbb{C})
$$


(typically extremely short, corresponding to the core curve of the solid torus). Make the attracting (resp. repelling) fixed point of $\varphi$ coincide with the North pole $P^{+}$(resp. the South pole $P^{-}$) of $\mathbb{S}^{2} \simeq \partial_{\infty} \mathbb{H}^{3}$; assume that $v$ lies on the Equator at longitude 0 , and orient the Equator along increasing longitudes. As a cover of the space $X$ which is triangulated, $U$ comes with a natural, $\varphi$-invariant decomposition into ideal tetrahedra.

The projection with respect to the center of Poincaré's ball model sends $\partial U$ homeomorphically to $\mathbb{S}^{2} \backslash\left\{P^{+}, P^{-}\right\} \backslash\left\{\varphi^{n}(v)\right\}_{n \in \mathbb{Z}}$. For each edge $v v^{\prime}$ of $\partial U$ (between ideal points $\left.v, v^{\prime} \in \mathbb{S}^{2}\right)$, this projection sends $v v^{\prime}$ to the short great-circle arc $\widehat{v v}^{\prime}$ in $\mathbb{S}^{2}$. If $v v^{\prime \prime}$ is another edge of $\partial U$, this allows us to speak about the angle $\widehat{v^{\prime} v v^{\prime \prime}} \in(-\pi, \pi]$ between $v^{\prime}$ and $v^{\prime \prime}$, as seen from $v$ (ie in $T_{v} \mathbb{S}^{2}$ ).

The punctured torus $\tau_{0}=\partial U / \varphi$ has three ideal edges, each endowed with a positive dihedral angle. Therefore the ideal vertex $v$ of $U$ is connected to six other vertices of $U$ by edges of $\partial U$, and there is a natural cyclic order on these six vertices. The equatorial plane intersects $\partial U$ along a broken line $J$ from $v$ to $v$ which is properly embedded in $\partial U$ (with both its endpoints ideal). We can orient $J$ along increasing longitudes.

Definition 18 Let $v_{1}, \ldots v_{6}$ (with indices seen modulo 6 ) denote the six neighbors of $v$ that are met, in that order, when turning counterclockwise around $v$, starting in the direction of the initial segment of $J$. For each $i$ in $\mathbb{Z} / 6 \mathbb{Z}$, there is an integer $n_{i} \in \mathbb{Z}$ such that $\varphi^{n_{i}}$ sends the following points to one another:

$$
\begin{aligned}
v_{i+2} & \mapsto v_{i+1} \\
\varphi^{n_{i}}: v_{i \pm 3} & \longmapsto v \\
v_{i-2} & \mapsto v_{i-1} .
\end{aligned}
$$

Of course, $n_{i}=-n_{i \pm 3}$. See Figure 8 .

Claim 19 The longitudes $l_{1}, l_{6}$ of $v_{1}$ and $v_{6}$ are both in $(0, \pi)$. The latitude of $v_{1}$ (resp. $\left.v_{6}\right)$ is positive (resp. negative).

Proof Since a half-turn around $v$ sends each $v_{i}$ to $v_{i+3}$, no angle $\widehat{v_{i-1} v v_{i}}$ in the tangent space to $\mathbb{S}^{2}$ at $v$ can exceed (or even reach) the value $\pi$; therefore $\widehat{v_{i-1} v v_{i}} \in$ $(0, \pi)$. Taking $i=1$, this proves the statement about latitudes. Therefore $v_{1}$ (resp. $v_{6}$ ) lies above (resp. below) the equatorial plane, and it also follows that $n_{6}<0<n_{1}$. Let $l_{i} \in(-\pi, \pi]$ denote the longitude of $v_{i}$ : clearly, $l_{i}<\pi$ since no edge of $\partial U$ can cross the North-South axis. The longitudes $l_{1}$ and $l_{6}$ cannot be both $\leq 0$, because 

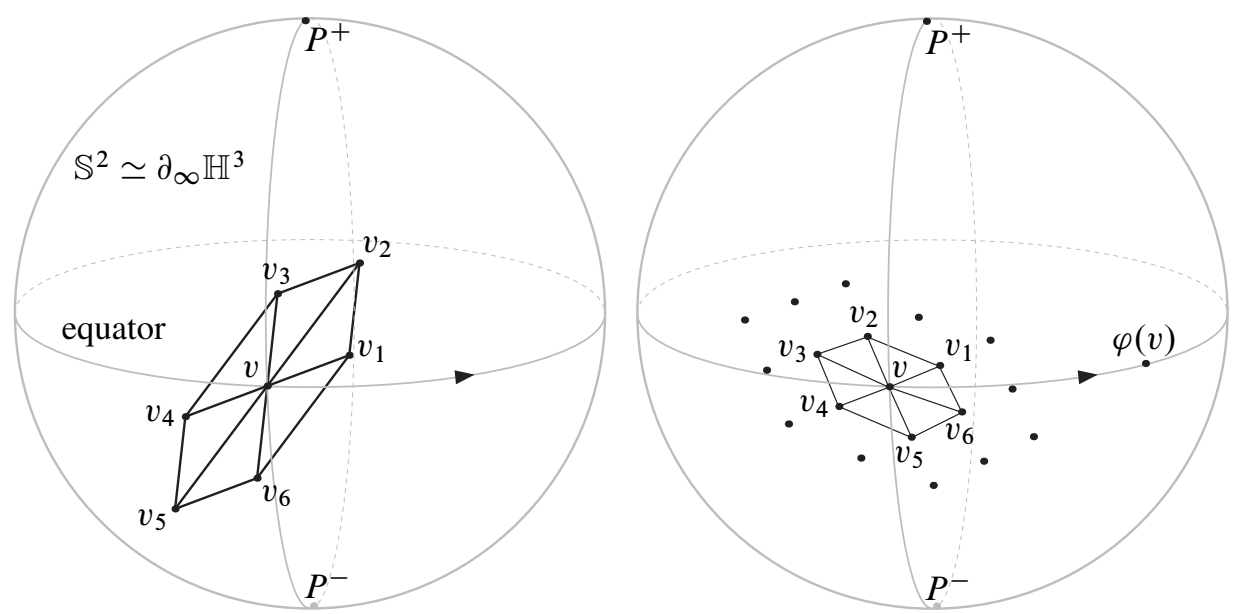

Figure 8: Left: One cannot have $l_{6} \leq 0<l_{1}$. Right: The actual situation (only some ideal vertices of $U$ are shown).

$\widehat{v_{6} v v_{1}} \in(0, \pi)$ and the East direction (increasing longitudes) lies between $v_{1}$ and $v_{6}$ as seen from $v$. Therefore, to show that $l_{1}, l_{6}$ are positive, we only need to assume

$$
l_{6} \leq 0<l_{1}
$$

and aim at a contradiction.

Note that on $\mathbb{S}^{2}$, for each $n>0$, the transformation $\varphi^{n}$ increases latitudes, and adds a constant angle to all longitudes (modulo $2 \pi$ ). Recall the relationships $v_{3}=\varphi^{-n_{6}}(v)$ and $v_{2}=\varphi^{n_{1}}\left(v_{3}\right)=\varphi^{-n_{6}}\left(v_{1}\right)$ : they imply that $v_{2}$ has highest latitude among $v_{1}, v_{2}, v_{3}$ (all three latitudes being positive; see the left panel of Figure 8). They also imply $l_{2} \equiv l_{1}-l_{6}(\bmod 2 \pi)$ : but $l_{2}$ cannot belong to $\left(\pi, l_{1}+\pi\right)+2 \pi \mathbb{Z}$ since the ideal triangle $v v_{1} v_{2} \subset \partial U$ cannot meet the North-South axis. Therefore, $l_{2}=l_{1}-l_{6}=l_{1}+l_{3}$ belongs to $\left(l_{1}, \pi\right)$, and the point $v_{2}$ also has the largest longitude among $v_{1}, v_{2}, v_{3}$, possibly tying with $v_{1}$ (and all three longitudes belong to $[0, \pi)$ ).

Let $v_{2}^{\prime}$ be the projection of $v_{2}$ to the Equator (along meridians), $v_{2}^{\prime \prime}$ the projection of $v_{2}$ to the zero meridian (along parallels), and consider the circle $\mathcal{C}$ through $v, v_{2}^{\prime}, v_{2}, v_{2}^{\prime \prime}$. By the latitude and longitude inequalities above, we see than $v_{1}, v_{3}$ both lie inside $\mathcal{C}$ on $\mathbb{S}^{2}$ (ie on the side of $\mathcal{C}$ that does not contain $P^{+}$and $P^{-}$). This contradicts the convexity of $U$ near the edge $v v_{2}$ : absurd. See Figure 8.

Remark 20 Claim 19 implies that $\varphi^{ \pm n_{1}}$ and $\varphi^{ \pm n_{6}}$ are, respectively, left- and righthanded. 
Recall the sequence of Farey triangles $p q r=T_{0}, T_{1}, \ldots, T_{N}=m s t$. The ideal edges $v v_{1}$ and $v v_{6}$ project (in $\partial X$ ) to the ideal arcs of slope $p$ and $q$ (up to order). Also, every $T_{i}$ for $i \geq 1$ has its vertices in the arc $\widehat{p q} \subset \mathbb{P}^{1} \mathbb{Q}$ that does not contain $r$ (in particular, the meridional slope $m$ belongs to that arc). Therefore, for every $i \in\{1,2, \ldots, N\}$ and every vertex $x$ of the Farey triangle $T_{i}$, we can draw a properly embedded intrinsic geodesic $g_{x}$ of slope $x$ in the punctured torus $\partial U / \varphi$ : this $g_{x}$ has a lift $\widehat{g_{x}} \subset \partial U$ that connects the ideal vertex $v$ to some $\varphi$-iterate of $v$, and whose initial (ideal) segment is contained in the ideal triangle $v v_{1} v_{6}$ of $\partial U$. We orient $\widehat{g_{x}}$ from $v$ to its other end. (As a particular case, $\widehat{g_{m}}$ is isotopic in $\partial U$ to the oriented equatorial curve $J$.)

Definition 21 When $x \in \mathbb{P}^{1} \mathbb{Q}$ is a vertex of some Farey triangle $T_{i}$ as above, define $v_{x} \in \mathbb{Z}$ as the integer such that the oriented curve $\widehat{g_{x}}$ runs from the ideal vertex $v$ to $\varphi^{v_{x}}(v)$.

We also define $\lambda_{x} \in \mathbb{R}$ as the integral of the longitude 1 -form in $\mathbb{S}^{2} \backslash\left\{P^{+}, P^{-}\right\}$along the closure of $\pi\left(\widehat{g_{x}}\right)$, where $\pi: \partial U \rightarrow \mathbb{S}^{2}$ is the central projection.

Proposition 22 Suppose $1 \leq i \leq N-1$ so that $T_{i}=a b c$ and $T_{i+1}=b c d$ are two consecutive Farey triangles. Then $v_{d}=v_{b}+v_{c}$ and $\lambda_{d}=\lambda_{b}+\lambda_{c}$.

Moreover, if $x \in \mathbb{P}^{1} \mathbb{Q}$ is a vertex of $T_{i}$ for some $i \in\{1, \ldots, N\}$, then $0<\lambda_{x} \leq 2 \pi$, with equality (for the upper bound) if and only if $x$ is the meridional slope $m$.

Proof Consider the ideal quadrilateral $Q:=(\partial U / \varphi) \backslash\left(g_{b} \cup g_{c}\right)$. The orientations of $g_{b}$ and $g_{c}$ induce orientations on the four edges of (the metric completion of) $Q$. Observe that $g_{d}$ runs diagonally across $Q$, from the vertex with two outgoing edges, to the vertex with two incoming edges: as a result, the closure of $\pi\left(\widehat{g_{d}}\right)$ in $\mathbb{S}^{2} \backslash\left\{P^{+}, P^{-}\right\}$is isotopic, with endpoints fixed, to the closure of

$$
\pi\left(\widehat{g_{b}} \cup \varphi^{v_{b}}\left(\widehat{g_{c}}\right)\right) \text { or, indifferently, of } \pi\left(\widehat{g_{c}} \cup \varphi^{v_{c}}\left(\widehat{g_{b}}\right)\right) \text {. }
$$

The exponent identity $v_{d}=v_{b}+v_{c}$ follows and, since $\varphi$ increases longitudes by a constant, so does the longitude identity $\lambda_{d}=\lambda_{b}+\lambda_{c}$.

By Claim 19, we have $\lambda_{p}, \lambda_{q} \in(0, \pi)$, so an immediate upward induction on $i$ now implies $\lambda_{x}>0$ for each vertex $x$ of $T_{i}$ (with $1 \leq i \leq N$ ). But $\lambda_{m}= \pm 2 \pi$, because the meridian curve $\widehat{g_{m}}$ runs exactly once around the infinite polyhedron $U$ : therefore, $\lambda_{m}=2 \pi$. Downward induction on $i$ finally yields $\lambda_{x}<2 \pi$ for $x \neq m$. 
Proposition 23 Suppose $1 \leq i \leq N-1$. Let $x \in \mathbb{P}^{1} \mathbb{Q}$ be the Farey vertex common to $T_{i-1}, T_{i}, T_{i+1}$. Then,

(i) one has $\lambda_{x} \in(0, \pi)$;

(ii) if the Farey triangle $T_{i}$ carries an $L$ (resp. an $R$ ), then $v_{x}>0$ (resp. $v_{x}<0$ );

(iii) if $T_{i}$ carries an $L$ (resp. an $R$ ), then $\varphi^{v_{x}}$ is left-handed (resp. right-handed).

Proof We name the vertices of the Farey triangles so that $T_{i}=x y z$ and $T_{i+1}=x z t$. By Proposition 22, one has $\lambda_{z}=\lambda_{x}+\lambda_{y}$ and $2 \pi \geq \lambda_{t}=\lambda_{x}+\lambda_{z}=2 \lambda_{x}+\lambda_{y}$. Since $\lambda_{x}, \lambda_{y}>0$, this yields (i).

Assertion (ii) follows from the following claim: if $l_{i}, r_{i} \in \mathbb{P}^{1} \mathbb{Q}$ are the left and right endpoints of the Farey edge $T_{i-1} \cap T_{i}$ (for the transverse orientation towards $m$ ), then $v_{r_{i}}<0<v_{l_{i}}$. This is clearly true for $i=1$ (in that case, $v_{l_{i}}=n_{1}$ and $v_{r_{i}}=n_{6}$, in the notation of Definition 18). For $i>1$, observe that

- one has $v_{m}=0$ because the curve $\widehat{g_{m}}$ is a closed curve around the ideal polyhedron $U$;

- by Proposition 22, the number $v_{m}$ is always a linear combination of $v_{l_{i}}$ and $v_{r_{i}}$ with positive integer coefficients;

- one has $v_{l_{i}} \neq 0$ and $v_{r_{i}} \neq 0$ because the curves $\widehat{g_{l_{i}}}$ and $\widehat{g_{r_{i}}}$ are not closed curves in $\partial U$.

These observations put together imply $v_{r_{i}}<0<v_{l_{i}}$ or $v_{l_{i}}<0<v_{r_{i}}$. The first is clearly the case by induction on $i$, because one always has $l_{i}=l_{i+1}\left(\right.$ resp. $\left.r_{i}=r_{i+1}\right)$ if the Farey triangle $T_{i}$ carries an $L$ (resp. an $R$ ).

Assertion (iii) is an immediate consequence of (i)-(ii).

\section{Canonical decomposition of a generic Dehn filling}

In this section we prove Theorem 1: to show that a given triangulation is Delaunay (or geometrically canonical), we essentially must prove a certain number of inequalities, which will boil down to statements of handedness as given by Proposition 23 .

Consider a hyperbolic manifold $M$ with $k \geq 2$ cusps, endowed with horoball neighborhoods, such that the genericity assumptions of Theorem 1 are satisfied. Let $\mathcal{D}$ denote the canonical triangulation of $M$. We assume that $H_{k}$, the horoball neighborhood of the $k$-th cusp $c_{k}$, has much smaller volume than some other $H_{i}$. 


\subsection{A generic small cusp}

First we prove that $\mathcal{D}$ contains exactly two ideal tetrahedra $\Delta, \Delta^{\prime}$ that have a vertex in $c_{k}$.

Consider a universal covering $\pi: \mathbb{H}^{3} \rightarrow M$ such that (in the upper half-space model) the point at infinity lies above the cusp $c_{k}$. Let $\Lambda$ be the rank-2 lattice of deck transformations of the form $z \mapsto z+\lambda$. Let $\left\{\eta_{i}\right\}_{i \in I}$ be the collection of all horoballs of $\mathbb{H}^{3}$ lying above some $H_{j}$ with $j<k$ (the $\eta_{i}$ are Euclidean balls tangent to the boundary $\mathbb{C}$ of the model half-space.) By the genericity assumption of Theorem 1 , there is a unique shortest path in $M$ from $H_{k}$ to $\bigcup_{j=1}^{k-1} H_{j}$ : therefore the largest $\eta_{i}$ (for the Euclidean metric) is unique modulo $\Lambda$.

We can assimilate $\Lambda$ to a lattice of $\mathbb{C}$, and assume that the largest $\eta_{i}$ 's are centered exactly at the points of $\Lambda$.

The Delaunay decomposition $D_{\Lambda}$ of $\mathbb{C}$ with respect to the vertex set $\Lambda$ consists either of isometric rectangles (all belonging to the same $\Lambda$-orbit), or of isometric triangles (belonging to two $\Lambda$-orbits) with strictly acute angles. We claim that the latter is the case: indeed, let $P \subset \mathbb{C}$ be a convex polygon of $D_{\Lambda}$ : the vertices of $P$, which are points of $\Lambda$, are on the boundary of a disk that contains no other points of $\Lambda$. Using the fact that the horoball $\eta_{\infty}$ centered at infinity stays very high above $\mathbb{C}$ in the half-space model (because $H_{k}$ has very small volume), it is easy to construct a ball of $\mathbb{H}^{3}$ that is tangent to the horoballs $\eta_{i}$ centered at the vertices of $P$, disjoint from all other $\eta_{i}$, and tangent to the horoball $\eta_{\infty}$. The center of this ball is a vertex of the Ford domain. Hence, there exists a cell of the Delaunay decomposition $\mathcal{D}$ of $M$ (more precisely, a lift $\widehat{\Delta}$ of such a cell to $\mathbb{H}^{3}$ ) whose vertices are exactly $\infty$ and the vertices of $P$. By the genericity assumption (I), $\widehat{\Delta}$ must be an ideal tetrahedron, so $P$ is a triangle, and has strictly acute angles. The two (isometric) $\Lambda$-orbits of triangles in the Delaunay decomposition $D_{\Lambda}$ of $\mathbb{C}$ correspond to two ideal tetrahedra $\Delta, \Delta^{\prime}$ in $\mathcal{D}$. Note that $\Delta \cup \Delta^{\prime}$ is a neighborhood of the cusp $c_{k}$.

The space $T=\partial\left(\Delta \cup \Delta^{\prime}\right) \subset M$ is the quotient by $\Lambda$ of the union of all ideal triangles of $\mathbb{H}^{3}$ that project vertically to triangles of $D_{\Lambda}$ (contained in $\mathbb{C}$ ): therefore, $T$ is a hyperbolic once-punctured torus bent along three lines, and its interior dihedral angles are twice those of $\Delta$ (or $\Delta^{\prime}$ ).

\subsection{Triangulation of the Dehn filling}

It is well-known that almost all (hyperbolic) Dehn fillings $M_{s}$ of $M$ at the cusp $c_{k}$ admit a spun decomposition $\mathcal{D}_{S}^{\text {spun }}$ into ideal, positively-oriented tetrahedra: namely, 
$\mathcal{D}_{s}^{\text {spun }}$ is obtained from $\mathcal{D}$ by letting the tips of $\Delta$ and $\Delta^{\prime}$ (formerly in $c_{k}$ ) spin asymptotically along the geodesic core of the filling solid torus of $M_{s}$ - actually, there are two such spun triangulations, spinning in opposite directions (see eg Thurston's notes [26, Chapter V]). Moreover, the cross-ratios of the tetrahedra of $\mathcal{D}_{s}^{\text {spun }}$ become (uniformly) close to those of $\mathcal{D}$ as the slope $s$ goes to infinity (ie "gets more and more complicated", eventually exiting any finite set). In particular, the punctured torus $T$, equal to the union of the bases of $\Delta$ and $\Delta^{\prime}$, is still embedded in $M_{S}$, with bending angles close to those in $M$.

Therefore, we can remove the solid torus $\overline{\Delta \cup \Delta^{\prime}}$ from the spun triangulation of $M_{S}$, and replace it with the solid torus $X$ constructed in Section 2 (with the same dihedral angles as $T$ ). By Proposition 8 (rigidity), $X$ is isometric to the closure of $\Delta \cup \Delta^{\prime}$, so after replacement we obtain a geometric ideal triangulation $\mathcal{D}_{S}$ of the filling $M_{S}$ (as in Theorem 1). In the remainder of Section 4 , we check that $\mathcal{D}_{s}$ is Delaunay.

\subsection{Minkowski space}

Our pictures (eg of the cusp link in Figure 6) are drawn in the upper half-space model of $\mathbb{H}^{3}$, but we will check geometric canonicity through a computation in the Minkowski space model. This section is only a quick reminder of the formulas relating the two models, and of Epstein and Penner's convex hull construction.

Endow $\mathbb{R}^{4}=\mathbb{R}^{3+1}$ with the Lorentzian product given by $\left\langle(x, y, z, t) \mid\left(x^{\prime}, y^{\prime}, z^{\prime}, t^{\prime}\right)\right\rangle:=$ $x x^{\prime}+y y^{\prime}+z z^{\prime}-t t^{\prime}$. Define

$$
\mathcal{X}:=\left\{v=(x, y, z, t) \in \mathbb{R}^{4} \mid t>0 \quad \text { and } \quad\langle v \mid v\rangle=-1\right\} .
$$

Then $\langle\cdot \mid \cdot\rangle$ restricts to a Riemannian metric on $\mathcal{X}$ and there is an isometry $\mathcal{X} \simeq \mathbb{H}^{3}$, with $\operatorname{Isom}^{+}(\mathcal{X})$ a component of $\mathrm{SO}_{3,1}(\mathbb{R})$. We will identify the point $(x, y, z, t)$ of $\mathcal{X}$ with the point at Euclidean height $1 /(t+z)$ above the complex number $(x+i y) /(t+z)$ in the Poincaré upper half-space model. Under this convention, the closed horoball $H_{d, \zeta}$ of Euclidean diameter $d$ centered at $\zeta=\xi+i \eta \in \mathbb{C}$ in the half-space model corresponds to $\left\{v \in \mathcal{X} \mid\left\langle v \mid v_{d, \zeta}\right\rangle \geq-1\right\}$, where

$$
v_{d, \zeta}=\frac{1}{d}\left(2 \xi, 2 \eta, 1-|\zeta|^{2}, 1+|\zeta|^{2}\right)
$$

We therefore identify the horoball $H_{d, \zeta}$ with the point $v_{d, \zeta}$ of the isotropic cone (check $\left.\left\langle v_{d, \zeta} \mid v_{d, \zeta}\right\rangle=0\right)$. Similarly, the closed horoball $H_{h, \infty}$ of points at Euclidean height no less than $h$ in the half-space model corresponds to $\left\{v \in \mathcal{X} \mid\left\langle v \mid v_{h, \infty}\right\rangle \geq-1\right\}$ where $v_{h, \infty}=(0,0,-h, h)$, so we identify $H_{h, \infty}$ with $v_{h, \infty}$.

Consider the following objects: a complete, oriented, cusped, finite-volume hyperbolic 3-manifold $M$, a horoball neighborhood $H_{c}$ of each cusp $c$, a universal covering 
$\pi: \mathbb{H}^{3} \rightarrow M$, and the group $\Gamma \subset \operatorname{Isom}^{+}\left(\mathbb{H}^{3}\right) \subset \mathrm{SO}_{3,1}(\mathbb{R})$ of deck transformations of $\pi$. The $H_{c}$ lift to an infinite family of horoballs $\left(H_{i}\right)_{i \in I}$ in $\mathbb{H}^{3}$, corresponding to a family of isotropic vectors $\left(v_{i}\right)_{i \in I}$ in Minkowski space, by the above construction. The closed convex hull $C$ of $\left\{v_{i}\right\}_{i \in I}$ in $\mathbb{R}^{3+1}$ is $\Gamma$-invariant, and its boundary $\partial C$ comes with a natural decomposition $\widetilde{\mathcal{D}}$ into polyhedral cells. In [11;3], Epstein, Penner and Akiyoshi proved:

Proposition 24 The simplicial complex $\widetilde{\mathcal{D}}$ defines a decomposition $\mathcal{D}$ of $M$ into convex ideal hyperbolic polyhedra, by projection of each face of $\widetilde{\mathcal{D}}$ to $\mathcal{X} \simeq \mathbb{H}^{3}$ (with respect to $0 \in \mathbb{R}^{3+1}$ ) and thence to $M$. The decomposition $\mathcal{D}$ of $M$ is dual to the Ford-Voronoi domain; $\mathcal{D}$ depends only on the mutual volume ratios of the $H_{c}$, but only a finite number of decompositions $\mathcal{D}$ arise as these volume ratios vary.

Conversely, given a decomposition $\mathcal{D}$ of the manifold $M$ (still endowed with the cusp neighborhoods $H_{c}$ ) into ideal polyhedra with vertices in the cusps, in order to prove that $\mathcal{D}$ is the Epstein-Penner decomposition, we only need to consider the decomposition $\widehat{\mathcal{D}}:=\pi^{*}(\mathcal{D})$ of $\mathbb{H}^{3}$ with vertex set the centers of the horoballs $\left\{H_{i}\right\}_{i \in I}$, lift $\hat{\mathcal{D}}$ to an infinite simplicial complex $\widetilde{\mathcal{D}}$ in Minkowski space $\mathbb{R}^{3+1}$ (the vertices $\left\{v_{i}\right\}_{i \in I}$ of $\widetilde{\mathcal{D}}$ lying over the $H_{i}$ in the isotropic cone, and the faces of $\widetilde{\mathcal{D}}$ being affine polyhedra), and show that $\widetilde{\mathcal{D}}$ is locally convex at each (co)dimension-2 face: indeed, the projection with respect to the origin provides a homeomorphism between $\mathcal{X} \simeq \mathbb{H}^{3}$ and $\mathcal{D} \backslash\left\{v_{i}\right\}_{i \in I}$; the disjoint union $\bigcup_{t \geq 1} t \tilde{D}$ is then automatically a convex body, and its faces are exactly the cells of $\tilde{D}$. In that case, we call $\mathcal{D}$ geometrically canonical.

Proposition 25 The codimension-one polyhedral complex $\widetilde{\mathcal{D}} \subset \mathbb{R}^{3+1}$, defined by a decomposition of $M$ into polyhedra, is locally convex if and only if for every 2dimensional facet $F=A_{1} \cdots A_{\sigma}$ of $\tilde{\mathcal{D}}$ (a planar polygon in $\mathbb{R}^{3+1}$ ), there exists a vertex $P \notin F$ of a 3 -dimensional face of $\widetilde{\mathcal{D}}$ containing $F$, and a vertex $Q \notin F$ of the other 3-dimensional face of $\tilde{\mathcal{D}}$ containing $F$, such that an identity of the form

$$
\rho P+(1-\rho) Q=\sum_{i=1}^{\sigma} \lambda_{i} A_{i} \text { where } \rho \in(0,1) \text { and } \sum_{i=1}^{\sigma} \lambda_{i}>1
$$

holds (some $\lambda_{i}$ 's can be negative, however).

Proof A more geometric way of stating the identity is as follows: if the hyperplane $\Pi \simeq \mathbb{R}^{3}$ is the linear span of the $A_{i}$ 's, then the affine span of the $A_{i}$ 's separates (in $\Pi$ ) the origin from the intersection of $\Pi$ with the segment $P Q$. This clearly expresses local convexity at the facet $A_{1} \cdots A_{\sigma}$, since $P$ and $Q$ are always on opposite sides of $\Pi$ (indeed their projections to $\partial_{\infty} \mathbb{H}^{3} \simeq \mathbb{S}^{2}$ are on opposite sides of the projection 
of $\Pi$ to $\mathbb{H}^{3}$ which is a plane). We express (12) by saying that $A_{1} \cdots A_{\sigma}$ lies below $P Q$ (as seen from the origin).

\subsection{Proving convexity in $\mathbb{R}^{3+1}$}

We now return to the ideal triangulation $\mathcal{D}_{s}$ of our Dehn filling, with the solid torus $X=\Delta_{1} \cup \cdots \cup \Delta_{N-1} \subset \mathcal{D}_{s}$. For each (triangular) face $F$ of $\mathcal{D}_{s}$ we must prove the convexity inequality (12) of Proposition 25 (applied to adjacent tetrahedra only, hence $\sigma=3)$.

If $F$ does not belong to $X$, recall that cross-ratios of tetrahedra outside $X$ in the filling $\mathcal{D}_{s}$ are close to what they were before filling in $\mathcal{D}$, while the volumes of the (remaining) cusp neighborhoods in the filled manifold $M_{s}$ are the same as in the unfilled manifold $M$ : therefore, the convexity inequality (12) in $\mathcal{D}_{s}$, for all but finitely many $s$, just follows from the analogous inequality in $\mathcal{D}$.

If $F$ is one of the two faces of $\partial X$, the inequality in $\mathcal{D}_{s}$ again follows from the geometric canonicity of $\mathcal{D}$. Indeed, check first that the two faces of $X$ are not glued to one another: if they were (by an orientation-reversing isometry), then the sum of angles around one of the three edges of $\partial X$ would be less than or equal to $\pi$. Therefore, the face $F$ separates a tetrahedron of $X$ from a tetrahedron outside $X$. Next, consider a cover $\pi: \mathbb{H}^{3} \rightarrow M$ sending infinity to $c_{k}$ (in the upper half-space model), and the induced decomposition $\hat{\mathcal{D}}:=\pi^{*}(\mathcal{D})$ of $\mathbb{H}^{3}$ into ideal tetrahedra. Consider a tetrahedron $\infty A B C$ of $\hat{\mathcal{D}}$, and the neighboring tetrahedron $A B C D$ (where $A, B, C, D \in \mathbb{C}$ and $A B C$ is an acute triangle whose circumscribed circle loops around $D$ ). Define $A^{\prime}:=B+C-A$, the symmetric image of $A$ with respect to the midpoint of $B$ and $C$, and similarly $B^{\prime}=A+C-B$ and $C^{\prime}=A+B-C$. The triangle $A B C$, together with $A^{\prime} B C$ (or $A B^{\prime} C$ or $A B C^{\prime}$ ), forms a fundamental domain of $\partial X$. Recall the tetrahedra of the solid torus $X$ are obtained by successive diagonal exchanges, beginning at the ideal triangulation of $\partial X$. If the very first diagonal exchange kills the edge $B C$ (resp. $C A$, resp. $A B$ ), the new edge must therefore be $A A^{\prime}$ (resp. $B B^{\prime}$, resp. $C C^{\prime}$ ). Hence, up to a permutation of $A, B, C$, the neighbor across $A B C$ of the tetrahedron corresponding (combinatorially) to $A B C D$ in $\mathcal{D}_{s}$, is the tetrahedron corresponding (combinatorially) to $A B C A^{\prime}$. Recall the infinite simplicial complex $\widetilde{\mathcal{D}} \subset \mathbb{R}^{3+1}$. If $a, b, c, d, a^{\prime}, f \in \mathbb{R}^{3+1}$ are the isotropic vectors lying above the horoballs centered at $A, B, C, D, A^{\prime}, \infty$ (respectively), then $a b c f$ and $a b c d$ are neighboring faces of $\widetilde{\mathcal{D}}$ (in particular, $a b c$ lies below the segment $f d$ as seen from the origin). But by convexity of $\widetilde{\mathcal{D}}$, the facet $a b c$ of $\widetilde{\mathcal{D}}$ also lies below any segment between vertices of $\widetilde{\mathcal{D}}$, provided this segment intersects the linear span of $a, b, c$. In particular, $a b c$ lies below $a^{\prime} d$ (because $A^{\prime}, D$ lie on opposite sides of 
the hyperbolic plane through $A, B, C$ ). This is still true for the lift $\widetilde{\mathcal{D}_{s}}$ of the filled triangulation $\mathcal{D}_{s}$ if the filling slope $s$ is chosen outside some finite set, because the cross-ratios in $\mathcal{D}_{s}$ are close to those in $\mathcal{D}$. Local convexity at the face $F=A B C$ of $\mathcal{D}_{s}$ is proved.

The only cases remaining are those when $F$ is an interior face of the solid torus $X$. We postpone to the end of the section the (easier) case of the "last" face, along which $\Delta_{N-1}$ is glued to itself, and focus on the other faces inside $X$.

Consider consecutive tetrahedra $\Delta_{i}$ and $\Delta_{i+1}$ of the filling solid torus of the manifold $M_{S}$, and their (adjacent) lifts $\Delta, \Delta^{\prime}$ in $\mathbb{H}^{3}$. We must check the convexity criterion of Proposition 25, the role of the 2-dimensional facet " $F$ " being played by the intersection of the lifts of $\Delta$ and $\Delta^{\prime}$ to $\mathbb{R}^{3+1}$.

We will assume that the letter $\Omega_{i}$ on the Farey triangle $T_{i}$ is an $L$ and proceed to a careful description of the cusp link, in Figure 9. Let us describe the figure.

- The top panel of Figure 9 shows a portion of the Farey graph; we name the Farey vertices $\alpha, \beta, \gamma, \delta, \epsilon$ so that $T_{i-1}=\alpha \beta \gamma, T_{i}=\alpha \gamma \delta, T_{i+1}=\alpha \delta \epsilon$ (enumerating the vertices of each triangle counterclockwise).

- The left (resp. right) panel shows four adjacent lifts of the ideal tetrahedron $\Delta_{i}$ (resp. $\left.\Delta_{i+1}\right)$ in $\mathbb{H}^{3}$. The vertices are ideal. The direction of the equator of $\mathbb{S}^{2} \simeq \partial_{\infty} \mathbb{H}^{3}$ is materialized by a grey line. The directions $\alpha, \beta, \gamma, \delta, \epsilon$ of some of the ideal edges are shown. The tetrahedra in the right panel lie glued behind the tetrahedra in the left panel; the triangulation in front of the right panel thus agrees with the triangulation in the back of the left panel. In each panel, the central ideal vertex $v$, assumed to lie on the equator, has been blown up (or truncated) to depict its link, which consists of four similar Euclidean triangles drawn in grey.

- The bottom panel puts these two ideal links together in one diagram consisting of three nested hexagons (we artificially draw each hexagon a tiny bit apart from the next one, even though they share four vertices). Each vertex of this figure corresponds to an ideal edge issued from $v$, and is marked with the slope $(\alpha, \beta, \gamma, \delta$ or $\epsilon)$ of that ideal edge. (Also compare these labels with the first panel of Figure 6.) The four triangles between two consecutive hexagons have the same triple of angles.

- The bottom panel equivalently represents, up to a similarity, the endpoints in $\mathbb{C}$ of ideal edges whose other endpoint is $\infty$ in the upper half-space model of $\mathbb{H}^{3}$ (the point $\infty$ corresponds to the central, blown-up vertex $v$ of the previous two panels). Each triangle of the bottom panel is the vertical projection to $\mathbb{C}$ of an ideal triangle of $\mathbb{H}^{3}$ which, once coned off to $\infty$, yields a tetrahedron of $\mathbb{H}^{3}$ isometric to $\Delta_{i}$ (outer triangles) or $\Delta_{i+1}$ (inner triangles). 


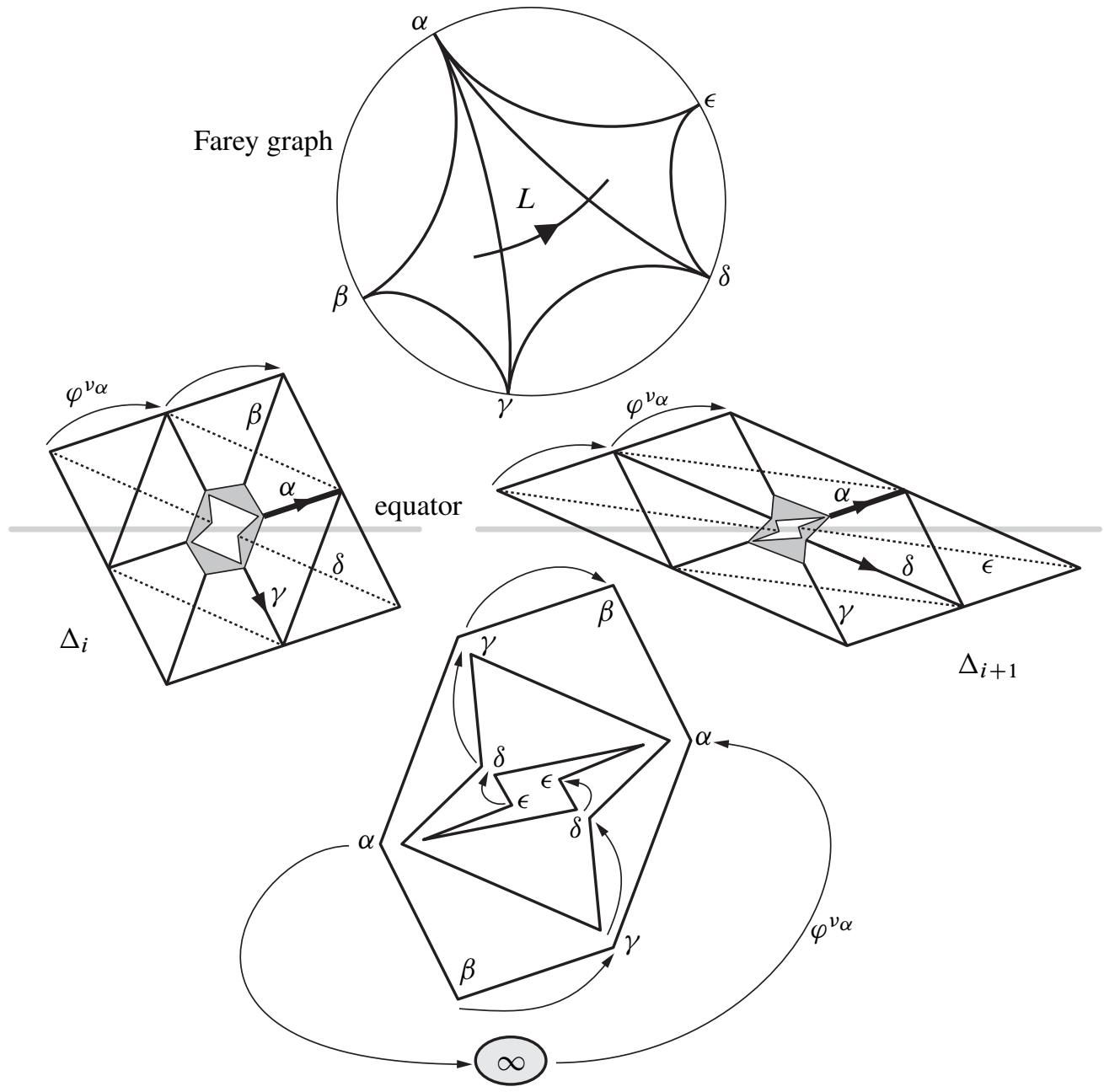

Figure 9: A "Left" in the Farey graph corresponds to a left-handed power of $\varphi$.

- In the left (resp. right) panel we have decorated edges of slope $\alpha$ and $\gamma$ (resp. $\alpha$ and $\delta$ ) with arrows. In the notation of Proposition 23, the loxodromy $\varphi^{v_{\alpha}}$ is left-handed (because $\Omega_{i}=L$ ). In these two panels, $\varphi^{v_{\alpha}}$ acts by sending the central vertex $v$ (tail of the edge marked $\alpha$ ) to the head of the edge marked $\alpha$, and by translating all other vertices along the same direction: for example, the head of the edge marked $\gamma$ goes to the head of the edge marked $\delta$.

This last observation allows us to understand the action of $\varphi^{v_{\alpha}}$ on the Riemann sphere $\mathbb{C} \cup\{\infty\}$ : in the bottom panel, where $v$ has been sent to $\infty$, the arrows indicate how $\varphi^{v_{\alpha}}$ acts on the vertices of the Euclidean hexagons (and $\infty$ ). For example, $\infty$ goes to 
a vertex marked $\alpha$ and the bottom-most vertex marked $\gamma$ goes to a vertex marked $\delta$. In the sequel, we must make sense of the left-handedness (Proposition 23) of this loxodromic action.

In order to shift to the "Minkowski space" aspect, we must take yet a closer look at the geometry of the link of the cusp (the following argument is taken from [16]). In the link of the cusp, up to a complex similarity, the link of the pleated surface $\tau_{i}$ between $\Delta_{i}$ and $\Delta_{i+1}$ is the centrally-symmetric hexagon $\left(-1, \zeta, \zeta^{\prime}, 1,-\zeta,-\zeta^{\prime}\right)$ in $\mathbb{C}$, as in Figure 10 (which reproduces the bottom panel of Figure 9): we assume that the vertices $-1,1$ both belong to the base segments of the Euclidean triangles just inside and just outside the hexagon.

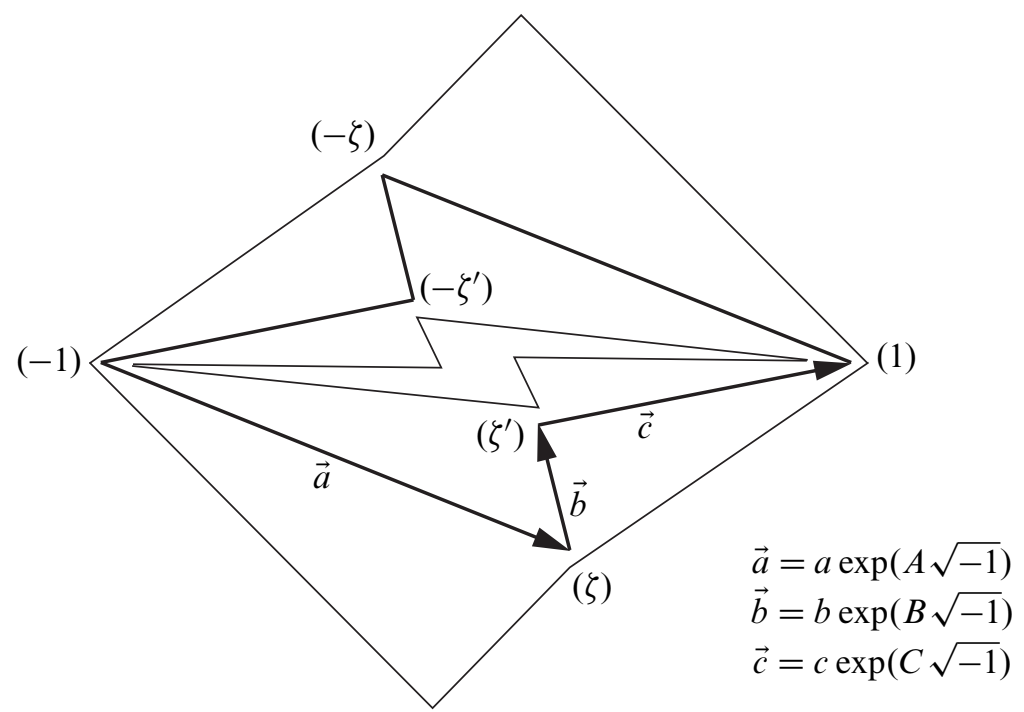

Figure 10: Adjacent tetrahedra $\Delta_{i}$ and $\Delta_{i+1}$ (cusp view). The hexagon corresponding to $\tau_{i}$ is in bold.

Let us introduce the notation

$$
\begin{aligned}
& \zeta+1=\vec{a}=a e^{i A} \\
& \zeta^{\prime}-\zeta=\vec{b}=b e^{i B} \\
& 1-\zeta^{\prime}=\vec{c}=c e^{i C}
\end{aligned}
$$

where $a, b, c \in \mathbb{R}^{>0}$ (so far $A, B, C$ are only defined modulo $2 \pi$ ). The map $f:=\varphi^{\nu_{\alpha}}$ now satisfies $f(-1)=\infty ; f(\infty)=1 ; f(\zeta)=\zeta^{\prime}$ : namely,

$$
f: u \mapsto 1+\frac{(\zeta+1)\left(\zeta^{\prime}-1\right)}{u+1}=1+\frac{\vec{a} \vec{c}}{u+1} .
$$


Therefore, using the notation $H_{\text {diameter, center }}$ for the horoballs of the upper half-space model (as in Section 4.3), we have $f\left(H_{1, \infty}\right)=H_{|\vec{a} \vec{c}|, f(\infty)}=H_{a c, 1}$. In other words, the Euclidean diameter of the horoball centered at the vertex 1 of the hexagon is $a c$, the product of the lengths of the adjacent edges of the hexagon. (By an easy argument, this relationship persists if the hexagon is scaled up or down, as long as the horoball centered at infinity is $H_{1, \infty}$.) For the same reason, the following horoballs are all sent to one another by deck transformations (in fact, by appropriate powers of $\varphi$ ):

$$
H_{1, \infty}, \quad H_{a c,-1}, \quad H_{a b, \zeta}, \quad H_{b c, \zeta^{\prime}}, \quad H_{a c, 1} .
$$

If $\zeta=\xi+\eta \sqrt{-1}$ and $\zeta^{\prime}=\xi^{\prime}+\eta^{\prime} \sqrt{-1}$, the isotropic vectors in Minkowski space corresponding to these horoballs are respectively, using (11):

$$
\begin{aligned}
& v_{\infty}=\left(\begin{array}{lllll}
0, & 0, & -1, & 1
\end{array}\right) \\
& v_{-1}=\frac{1}{a c}(-2,0, \quad 0, \quad 2 \quad) \\
& v_{\zeta}=\frac{1}{a b}\left(2 \xi, \quad 2 \eta, 1-|\zeta|^{2}, 1+|\zeta|^{2}\right) \\
& v_{\zeta^{\prime}}=\frac{1}{b c}\left(2 \xi^{\prime}, 2 \eta^{\prime}, 1-\left|\zeta^{\prime}\right|^{2}, 1+\left|\zeta^{\prime}\right|^{2}\right) \\
& v_{1}=\frac{1}{a c}\left(\begin{array}{llll}
2, & 0, & 0, & 2
\end{array}\right) \text {. }
\end{aligned}
$$

To check the convexity criterion of Proposition 25 at the codimension-two face (in $\mathbb{R}^{3+1}$ ) projecting to $\left(\zeta \zeta^{\prime} \infty\right)$, it is enough to show that if $\lambda v_{\zeta}+\mu v_{\zeta^{\prime}}+v v_{\infty}=$ $\rho v_{1}+(1-\rho) v_{-1}$ then $\lambda+\mu+v>1$ (moreover, this will in fact take care of both faces along which $\Delta_{i}$ touches $\Delta_{i+1}$ in the filling solid torus $X$ ). One easily finds the unique solution

$$
\lambda=\frac{b \eta^{\prime}}{c\left(\eta^{\prime}-\eta\right)}, \quad \mu=\frac{-b \eta}{a\left(\eta^{\prime}-\eta\right)}, \quad v=\frac{\eta^{\prime}\left(1-|\zeta|^{2}\right)-\eta\left(1-\left|\zeta^{\prime}\right|^{2}\right)}{a c\left(\eta^{\prime}-\eta\right)}
$$

(we will not need the value of $\rho$ ), hence

$\lambda+\mu+v=1+\frac{Z}{a c\left(\eta^{\prime}-\eta\right)}$ where $Z=a b \eta^{\prime}-b c \eta+\eta^{\prime}\left(1-|\zeta|^{2}\right)-\eta\left(1-\left|\zeta^{\prime}\right|^{2}\right)-a c\left(\eta^{\prime}-\eta\right)$

Observe that $\eta^{\prime}>\eta$ because the triangles $-1 \zeta \zeta^{\prime}$ and $1 \zeta^{\prime} \zeta$ are counterclockwise oriented. So it is enough to prove that $Z>0$. Endow $\mathbb{C} \simeq \mathbb{R}^{2}$ with the usual scalar product, denoted " $\diamond$ " to avoid confusion with scalar multiplication, and observe that 


$$
\begin{aligned}
1 & -|\zeta|^{2}=\vec{a} \diamond(\vec{b}+\vec{c}) \text { and } 1-\left|\zeta^{\prime}\right|^{2}=(\vec{a}+\vec{b}) \diamond \vec{c} \text {. Hence } \\
Z & =\eta^{\prime}(a b+\vec{a} \diamond \vec{b})-\eta(b c+\vec{b} \diamond \vec{c})-\left(\eta^{\prime}-\eta\right)(a c-\vec{a} \diamond \vec{c}) \\
& =a b c\left(\frac{\eta^{\prime}}{c}(1+\cos (A-B))-\frac{\eta}{a}(1+\cos (B-C))-\frac{\eta^{\prime}-\eta}{b}(1-\cos (A-C))\right) \\
& =-a b c(\sin C(1+\cos (A-B))+\sin A(1+\cos (B-C))+\sin B(1-\cos (A-C))) \\
& =-4 a b c \sin \frac{A+C}{2} \cos \frac{B-A}{2} \cos \frac{B-C}{2}
\end{aligned}
$$

by standard trigonometric formulae. Observe that the last expression is a well-defined function of $A, B, C \in \mathbb{R} / 2 \pi \mathbb{Z}$ (although each factor is defined only up to sign). Next, however, we will be careful which representatives of $A, B, C$ in $\mathbb{R}$ we pick. First, we choose for $B$ the smallest positive representative. Since the triangles $-1 \zeta \zeta^{\prime}$ and $1 \zeta^{\prime} \zeta$ are counterclockwise oriented, it follows that $B \in(0, \pi)$ and we can pick $A, C$ in $(B-\pi, B)$. Since $\vec{a}+\vec{b}+\vec{c}=2$ must also have an argument in $(B-\pi, B)$, one necessarily has

$$
-\pi<\min \{A, C\}<0<B<\pi \text { and } A, C \in(B-\pi, B) .
$$

In particular, to prove that $Z>0$, it only remains to show that

$$
-\pi<\frac{A+C}{2}<0 \text {. }
$$

For the deck transformation $f: u \mapsto 1+\vec{a} \vec{c} /(u+1)$ studied above, Definition 17 yields hand $(f)=4 /(\vec{a} \vec{c})$. But $f$ is left-handed by Proposition 23 , so $\operatorname{Im}(\vec{a} \vec{c})<0$ ie $A+C \in(-\pi, 0)+2 \pi \mathbb{Z}$. By (15), we have $-2 \pi<A+C<\pi$ a priori, hence in fact $-\pi<A+C<0$. Therefore (16) must hold. Geometric canonicity at the interface of tetrahedra $\Delta_{i}$ and $\Delta_{i+1}$ is proved (the argument is similar if the Farey triangle $T_{i}$ carries an $R$ instead of an $L$ ).

It remains to prove geometric canonicity at the core of the filling solid torus itself, where the last tetrahedron $\Delta_{N-1}$ is glued to itself along an ideal triangle. The "hexagon" $H_{N-1}$ of $\mathbb{C}$ has two opposite interior angles equal to 0 and is therefore collapsed to a broken line of three segments. In (14) (and Figure 10), this simply translates as the identity $\zeta^{\prime}=-1$; the collapsed hexagon is the broken line $(\zeta,-1,1,-\zeta)$. The radii of the horoballs centered at these vertices are computed exactly as in (13), under the extra assumption $\zeta^{\prime}=-1$.

The tetrahedra with ideal vertices $(\infty, 1,-1, \zeta)$ and $(\infty, 1,-1,-\zeta)$ are glued along the face $(\infty, 1,-1)$, and the isotropic vectors in Minkowski space corresponding to 
their vertices are, following (14):

$$
\begin{aligned}
& v_{\infty}=\left(\begin{array}{llll}
0, & 0, & -1, & 1
\end{array}\right) \\
& v_{1}=\frac{1}{2|1+\zeta|}(2, \quad 0, \quad 0, \quad 2 \quad) \\
& v_{-1}=\frac{1}{2|1+\zeta|}\left(\begin{array}{cccc}
-2, & 0, & 0, & 2
\end{array}\right) \\
& v_{\zeta}=\frac{1}{|\zeta+1|^{2}}\left(\begin{array}{ccc}
2 \xi & 2 \eta, & 1-\xi^{2}-\eta^{2}, 1+\xi^{2}+\eta^{2}
\end{array}\right) \\
& v_{-\zeta}=\frac{1}{|\zeta+1|^{2}}\left(-2 \xi,-2 \eta, 1-\xi^{2}-\eta^{2}, 1+\xi^{2}+\eta^{2}\right) \text {. }
\end{aligned}
$$

The equation $\rho v_{\zeta}+(1-\rho) v_{-\zeta}=\lambda v_{\infty}+\mu v_{1}+\nu v_{-1}$ has a unique solution, namely $\rho=1 / 2$ and

$$
\lambda=\frac{|\zeta|^{2}-1}{|\zeta+1|^{2}} \quad \text { and } \quad \mu=v=\frac{1}{|\zeta+1|}
$$

Clearly, one will have $\lambda+\mu+v>1$ if and only if $|\zeta|^{2}-1+2|\zeta+1|>|\zeta+1|^{2}$, or equivalently, $|\zeta|^{2}>(|\zeta+1|-1)^{2}$ : but this relationship follows from the triangular inequality in the Euclidean triangle $(0,-1, \zeta)$. Therefore, by Proposition 25, the convexity inequality in Minkowski space is satisfied. Theorem 1 is proved.

\subsection{Filling on several cusps}

An analogue of Theorem 1 holds when several cusps undergo Dehn filling. The genericity assumptions (I)-(II), however, must be suitably extended.

Let $M$ be a complete hyperbolic 3-manifold with cusps $c_{1}, \ldots, c_{k}$, endowed with horoball neighborhoods $H_{1}, \ldots, H_{k}$ (where $k \geq 2$ ). Let $\ell$ be an integer, $1<\ell \leq k$. Make the following assumptions:

(I) The decomposition $\mathcal{D}$ (before filling) consists only of ideal tetrahedra.

(II) For each integer $j$ such that $\ell \leq j \leq k$, there exists a unique shortest path from $H_{j}$ to $\bigcup_{i=1}^{\ell-1} H_{i}$ in $M$.

Theorem 26 Under the assumptions (I)-(II) above, if the volumes of the neighborhoods $H_{\ell}, \ldots, H_{k}$ are much smaller than one of $H_{1}, \ldots, H_{\ell-1}$, then for each integer $j$ such that $\ell \leq j \leq k$, the canonical decomposition $\mathcal{D}$ of $M$ (before filling) contains exactly two tetrahedra $\Delta_{j}, \Delta_{j}^{\prime}$ with a vertex in the cusp $c_{j}$; the tetrahedra $\Delta_{j}$ and $\Delta_{j}^{\prime}$ are isometric and have each exactly one vertex in $c_{j}$ and three vertices in $\bigcup_{i=1}^{\ell-1} c_{i}$.

Moreover, for each $\ell \leq j \leq k$ there exists a finite set of slopes $\mathcal{X}_{j}$ in the cusp $c_{j}$ such that for any choice of slopes $s_{\ell}, \ldots, s_{k}$ in $c_{\ell}, \ldots, c_{k}$ satisfying $s_{j} \notin \mathcal{X}_{j}$ for each $j$, the 
canonical decomposition $\mathcal{D}_{s}$ obtained by Dehn filling along the slopes $s_{\ell}, \ldots, s_{k}$ is combinatorially of the form

$$
\mathcal{D}_{s}=\left(\mathcal{D} \backslash \bigcup_{j=\ell}^{k}\left\{\Delta_{j}, \Delta_{j}^{\prime}\right\}\right) \cup \bigcup_{j=\ell}^{k} \mathcal{T}_{j}
$$

where $\mathcal{T}_{j}=\left\{\Delta_{1}^{(j)}, \ldots, \Delta_{N_{j}-1}^{(j)}\right\}$ is a triangulation of a solid torus minus one boundary point, and the combinatorial gluing of the $\Delta_{i}^{(j)}$ (for $j$ fixed) is dictated by the continued fraction expansion of the slope $s_{j}$, with respect to a basis of the first homology of the cusp $c_{j}$ depending only on $\mathcal{D}$.

In other words, as long as the cusp neighborhoods $H_{\ell}, \ldots, H_{k}$ are small enough and the slopes $s_{\ell}, \ldots, s_{k}$ are long enough, Theorem 1 applies "simultaneously" to all cusps $c_{\ell}, \ldots, c_{k}$. The proof of Theorem 1 transposes without major changes to Theorem 26, using the multicusped version of Thurston's hyperbolic Dehn surgery theorem (see eg Theorem 5.8.2 and the discussion immediately following it in [26]).

As a corollary, if (I) and (II) are satisfied and the horoballs $H_{\ell}, \ldots, H_{k}$ are small enough compared to one of $H_{1}, \ldots, H_{\ell-1}$, then any sufficiently long filling of some of $c_{\ell}, \ldots, c_{k}$ is generic with respect to the surviving unfilled cusps among $c_{\ell}, \ldots, c_{k}$.

\section{Fillings of the Whitehead link complement}

In this section we describe the Delaunay decompositions of all hyperbolic Dehn fillings of one cusp of the Whitehead link complement.

\subsection{Canonical decomposition before filling}

The following facts are classical; we refer to [26] or to Weeks' program SnapPea [27] for further background. More material on the Whitehead link can be found in [22].

Let $A B C D$ and $D C B^{\prime} A^{\prime}$ be two adjacent unit squares of $\mathbb{C}$ (vertices enumerated clockwise and belonging to $\mathbb{Z}[i]$, as in Figure 11). Let $Q, Q^{\prime}$ be the convex hulls of $\infty, A, B, C, D$ and of $\infty, D, C, B^{\prime}, A^{\prime}$ respectively, taken in the upper half-space model of $\mathbb{H}^{3}$. Then $Q \cup Q^{\prime}$ is a fundamental domain of the hyperbolic Whitehead link complement $M$ (census manifold $\mathrm{m} 129$ ); the face identifications are the translations of vector $\overrightarrow{A B}=i, \overrightarrow{A A^{\prime}}=2$, and the hyperbolic isometry sending $A, B, C, D$ to $D, A^{\prime}, B^{\prime}, C$ respectively. Let $c_{1}, c_{2}$ be the two cusps of $M$, with $c_{2}$ being the cusp at infinity. Note that the decomposition $Q \cup Q^{\prime}=M$ is the Delaunay decomposition of $M$ when the horoball neighborhood of $c_{2}$ has volume less than half that of $c_{1}$. 
Note that $M$ has isometries that exchange $c_{1}$ and $c_{2}$, but has no orientation-reversing isometries (so the Whitehead link is chiral).

Note also that the decomposition $Q \cup Q^{\prime}$ of $M$ does not satisfy the first and second "genericity" assumptions of Theorem 1: the cells are not tetrahedra, and the horoballs centered at $B$ and $C$, while belonging to different orbits of the stabilizer $2 \mathbb{Z} \oplus i \mathbb{Z}$ of $\infty$ in the group of deck transformations, are at the same distance from $c_{2}$. Thus, Theorem 1 does not apply directly.

As a bit of notation: if $k, l$ are coprime integers, let $s=l / k$ denote the slope in the cusp $c_{2}$ represented by the vector $k \overrightarrow{A A^{\prime}}+l \overrightarrow{A B}$. That is, we choose the shortest possible basis for $H_{1}\left(c_{2}, \mathbb{Z}\right)$. (The census manifold $\mathrm{m} 129$ uses the same basis up to order, but as the complement of the link of Figure 11, it has link-theoretic meridian $\overrightarrow{A B}$ and longitude $\overrightarrow{A A^{\prime}}-2 \overrightarrow{A B}$. This is not canonical since many links in $\mathbb{S}^{3}$ have complement m129.) Let $M_{S}=\operatorname{m} 129(l, k)$ be the manifold obtained by filling $c_{2}$ along the slope $s$. The following result is a consequence of Theorem 1.2 of [21].

Proposition 27 The Dehn filling $M_{s}$ is hyperbolic if and only if

$$
\pm(k, l) \notin\{(0,1),(1,0),(1, \pm 1),(1, \pm 2)\} .
$$

In the remainder of this section we assume $(k, l)$ satisfies the condition of Proposition 27 and adapt the argument of Sections 1-4 to describe the Delaunay decomposition of $M_{S}$ (thus reproving, in particular, the "if" direction). This decomposition will always consist in replacing $Q \cup Q^{\prime} /\langle z \mapsto z+2, z \mapsto z+i\rangle$ with a triangulated solid torus $Y$ whose exterior faces are two (triangulated) ideal quadrilaterals, which we then identify.

\subsection{First case: $l$ is odd}

If $l$ is odd, then the vector $k \overrightarrow{A A^{\prime}}+l \overrightarrow{A B}=2 k+i l \in \mathbb{C}$ is irreducible in the lattice $\mathbb{Z}[i]$. For that reason, we can take for $Y$ the double cover of the solid torus $X$ constructed in Section 2.

More precisely, let $m \in \mathbb{P}^{1} \mathbb{Q}$ be the Farey vertex $l /(2 k)$ (irreducible fraction). Then $m$ does not belong to $\left\{0, \pm 1, \pm 2, \pm \frac{1}{2}, \infty\right\}$ : the first three are ruled out because $m$ has even denominator; the last two because we assumed $\pm(k, l) \notin\{(0,1),(1, \pm 1)\}$. According to the value of $m$, choose $(p, q, r)$ as follows:

$$
\begin{array}{r||c|c|c|c}
\text { if } & m<-2 & -2<m<-1 & -1<m<-1 / 2 & -1 / 2<m<0 \\
(p, q, r)= & (\infty,-1,0) & (-1, \infty, 0) & (-1,0, \infty) & (0,-1, \infty) \\
\hline \text { if } & 0<m<1 / 2 & 1 / 2<m<1 & 1<m<2 & 2<m \\
(p, q, r)= & (0,1, \infty) & (1,0, \infty) & (1, \infty, 0) & (\infty, 1,0)
\end{array}
$$



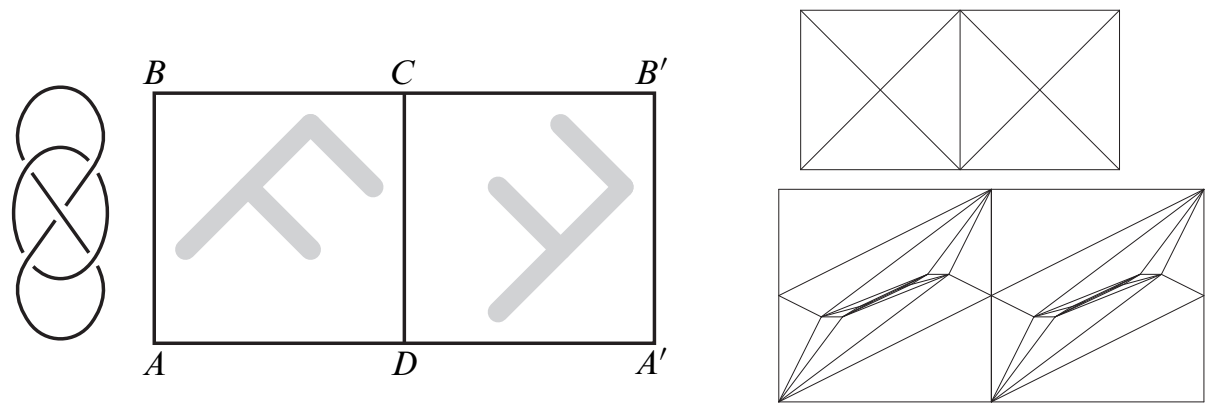

Figure 11: Left: The (chiral) Whitehead link m129. Middle: Cusp view of m129 from the common tip of the square-based pyramids $Q$ and $Q^{\prime}$, ie from the cusp that will be filled. The $F$-shaped symbol drawn on the bases of $Q$ and $Q^{\prime}$ shows their identification. Right: View of the canonical decomposition from the other cusp of $\mathrm{m} 129$, before (top) and after (bottom) a Dehn filling with $(k, l)=(11,8)$. In the top panel, the centers of the two squares project to the cusp that will be filled. In the bottom panel, we see that the tetrahedra in the decomposition of the filling become very close to flat, very quickly.

The relative positions of $p, q, r, m$ are then exactly as in Section 2: namely, $p q$ separates $r$ from $m$; the point $m$ is not the other common Farey neighbor $r^{\prime}$ of $p$ and $q$; and the line $r m$ crosses $p r^{\prime}$ (not $q r^{\prime}$ ). In particular, using the wedge notation (4) one has $m \wedge r \geq 3$.

Let $\theta \in(0, \pi)$ be a parameter and define

$$
\left(\theta_{p}, \theta_{q}, \theta_{r}\right):=\left\{\begin{array}{l}
(0, \theta, \pi-\theta) \text { if } p= \pm 1 \text { ie }|m| \in(1 / 2,2) ; \\
(\theta, 0, \pi-\theta) \text { if } q= \pm 1 \text { ie }|m| \notin(1 / 2,2) .
\end{array}\right.
$$

This choice will cause the "diagonal" edges of slope \pm 1 to be flat, while the edges of slope 0 and $\infty$ will be bent. Since $m \wedge r \geq 3$, it is straightforward to check that $\left(\theta_{p}, \theta_{q}, \theta_{r}\right)$ satisfies the hypothesis $(m \wedge p) \theta_{p}+(m \wedge q) \theta_{q}+(m \wedge r) \theta_{r}>2 \pi$ of Proposition 10 if and only if $\theta$ belongs to some subinterval $\Theta=\left(0, \theta_{\max }\right) \subset(0, \pi)$.

Apply now Proposition 10 and Corollary 16 with $\theta \in \Theta$. We obtain an ideal hyperbolic solid torus $X$ with dihedral angles $\theta, 0, \pi-\theta$. Let $P$ be the fundamental domain of $\partial X$ defined as the ideal quadrilateral cut out by the edges of slope 0 and $\infty$. Let $Y$ be the double cover of $X$. Since the meridian slope is $m=l /(2 k)$ and the determinant

$$
\left|\begin{array}{cc}
1 & l \\
0 & 2 k
\end{array}\right|
$$


is even, the curve of slope $1 / 0=\infty$ in $\partial X$ is homotopic to an even power of the core, and therefore lifts to a closed curve in $Y$, while the curve of slope $0 / 1=0$ does not (because

$$
\left|\begin{array}{cc}
0 & l \\
1 & 2 k
\end{array}\right|
$$

is odd). Therefore, a fundamental domain of $\partial Y$ is obtained by gluing two copies $P, P^{\prime}$ of the ideal quadrilateral $P$ side by side along the edge of slope $\infty$. We view $P, P^{\prime}$ as immersed in the twice-punctured torus $\partial Y$.

We now glue $P$ to $P^{\prime}$ by an orientation-reversing isometry, in the same way the square bases of the pyramids $Q, Q^{\prime}$ were glued together to yield the Whitehead link complement $M$ (Figure 11, left). By construction, the quotient of $Y$ under this identification is homeomorphic to the Dehn filling $M_{S}$. The angular part of the gluing equation is automatically satisfied, since the two flat edges of $\partial Y$ (diagonals of $P, P^{\prime}$ ) are identified, and all four nonflat edges of $\partial Y$ are identified to one edge at which the sum of dihedral angles is $\theta+(\pi-\theta)+\theta+(\pi-\theta)=2 \pi$.

Therefore, the space $W$ of angle structures associated to our triangulation of $M_{S}$ (as in Theorem 5) is described by setting $\left(\theta_{p}, \theta_{q}, \theta_{r}\right)$ as in (18) and finding all $\left(\theta_{p}, \theta_{q}, \theta_{r}\right)-$ angle structures in the sense of Proposition 10 as $\theta$ varies freely in $\Theta \subset(0, \pi)$.

Proposition 28 The volume functional has a critical point, namely a maximum, on $W$.

Proof Exactly as in Proposition 15, the maximum of the (extended) volume functional is achieved at some point $z=\left(z_{i}\right)_{0 \leq i \leq N}$ of the closure of $W$. Using (18), the system of constraints (7) satisfied by $z$ now becomes

or

$$
\begin{aligned}
& \left(z_{0}, z_{1}, z_{2}, \ldots, z_{N-1}, z_{N}\right) \\
& =\left(\pi, \theta, z_{2}, \ldots, z_{N-1}, 0\right) \\
& \left(\pi+\theta, \theta, z_{2}, \ldots, z_{N-1},\right.
\end{aligned}
$$

according to whether $|m| \in(1 / 2,2)$ or not.

In the first case, suppose $\theta=\pi$. By the convexity condition of (10), one then has $z_{0}=z_{1}=\cdots=z_{h}=\pi$ where $\Delta_{h}$ is the first hinge tetrahedron. The hinge condition of (10) then implies $z_{h-1} \geq z_{h}+z_{h+1}$, hence $z_{h+1}=0$. That in turn implies $z_{i}=0$ for all $i>h$ (we observed in the proof of Proposition 15 that the sequence $\left(z_{i}\right)$ is nonincreasing). Therefore all tetrahedra $\Delta_{i}$ are flat, and the volume is certainly not maximal. 
In the second case, suppose $\theta=\pi$. Table (9) implies $\pi-\left(z_{0}+z_{2}\right) / 2 \geq 0$ hence $z_{2}=0$ and $z_{i}=0$ for all $i>1$ : again, all $\Delta_{i}$ are flat, so the volume is certainly not maximal.

Therefore, $\theta<\pi$. The argument of Proposition 15 now follows through unchanged to show that no parameter $z_{i}$ for $0<i<N$ belongs to $\{0, \pi\}$. By Proposition 7, all tetrahedra $\Delta_{i}$ have only positive angles (ie $z \in W$ ).

Theorem 5 applies: we have found a complete hyperbolic structure on the triangulated space $M_{S}$. To check that the triangulation is canonical, we only need to check the Minkowski convexity relationship (12). For interior faces of the solid torus $Y$, this is already done (Section 4.4). For the boundary faces, we must describe more precisely the cusp triangulation of $M_{S}$.

Each of the two ideal vertices of the solid torus $Y$ (projecting to the single ideal vertex of $X$ ) has a cusp triangulation made of nested, centrally symmetric hexagons (as in Figure 6, right). However, by (18), two opposite angles of the outermost hexagon $H_{0}$ are equal to $\pi$, so the general cusp shape is a 4-sided parallelogram. Moreover, the edges $v v^{\prime}, v v^{\prime \prime}$ of $H_{0}$ adjacent to a flat vertex $v$ have the same length: indeed, the ideal quadrilateral $\infty v^{\prime} v v^{\prime \prime}$ must be a square (ie its diagonals cross at a right angle), because it is a face of $Y$ and the gluing of the two isometric faces of $Y$ that yields the Dehn filling $M_{S}$ sends horizontal edges of one face to vertical edges of the other (eg as in Figure 11).

The universal cover of the cusp triangulation of $M_{S}$ is a union of translated copies of the cusp triangulation of $Y$. For example, up to a plane similarity, the outermost hexagons in two adjacent translates can be taken to be (for some $\zeta \in \mathbb{C} \backslash \mathbb{R}$ )

and

$$
(2 \zeta-1, \zeta-1,-1,1, \zeta+1,2 \zeta+1)
$$$$
(-2 \zeta-1,-\zeta-1,-1,1,-\zeta+1,-2 \zeta+1)
$$

so the cusp triangles $(-1,1, \zeta+1)$ and $(-1,1,-\zeta-1)$ share an edge $(-1,1)$. We apply Proposition 25 to the ideal triangle $(\infty, 1,-1)$ - by symmetry this will deal with all four triangular faces of the solid torus $Y$ (note that for proving the Minkowski convexity relationship (12), we do not care whether or not the two adjacent hexagons above are in the same orbit of the stabilizer of $\infty$ ).

Following the method of Section 4.4 (especially (13) and the discussion that precedes it), if $\zeta=\xi+i \eta$, the isotropic vectors in $\mathbb{R}^{3+1}$ corresponding to the horoballs centered 
at $\infty, 1,-1, \zeta+1,-\zeta-1$ are respectively

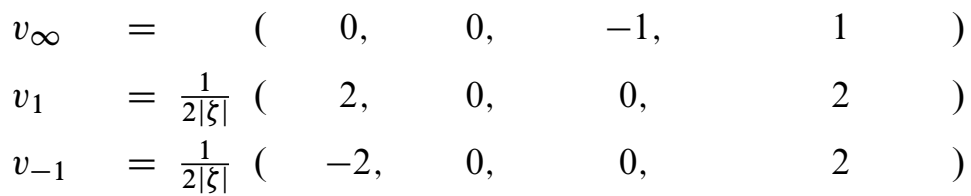

$$
\begin{aligned}
& v_{\zeta+1}=\frac{1}{|\zeta|^{2}}\left(2 \xi+2, \quad 2 \eta, \quad 1-|\zeta+1|^{2}, 1+|\zeta+1|^{2}\right) \\
& v_{-\zeta-1}=\frac{1}{|\zeta|^{2}}\left(-2 \xi-2,-2 \eta, 1-|\zeta+1|^{2}, 1+|\zeta+1|^{2}\right) \text {. }
\end{aligned}
$$

The solution to $\rho v_{\zeta+1}+(1-\rho) v_{-\zeta-1}=\lambda v_{1}+\mu v_{\infty}+v v_{-1}$ satisfies

$(\lambda, \mu, v)=\left(\frac{1}{|\zeta|}, \frac{|\zeta+1|^{2}-1}{|\zeta|^{2}}, \frac{1}{|\zeta|}\right), \quad$ hence $\lambda+\mu+v=1+\frac{|\zeta+1|^{2}-(|\zeta|-1)^{2}}{|\zeta|^{2}}>1$ according to the triangular inequality in the triangle $(0, \zeta,-1)$ : by Proposition 25, the convexity inequality in Minkowski space is satisfied.

\subsection{Second case: $l$ is even}

If $l$ is even, then the vector $k \overrightarrow{A A^{\prime}}+l \overrightarrow{A B}=2 k+i l \in \mathbb{C}$ is twice the irreducible vector $k+i(l / 2)$ in the lattice $\mathbb{Z}[i]$. For that reason, the ideal solid torus $Y$ cannot be taken to be simply a cover of $X$. Instead, we must introduce a variant of the construction of Section 2. To give a preview of the difference with Section 2, if $U \subset \mathbb{H}^{3}$ is a universal cover of the solid torus $Y$ we will construct below and $\langle\varphi\rangle \simeq \mathbb{Z}$ is the group of deck transformations of $U$, then for each ideal vertex $v$ of $U$, the symmetric image $v^{\prime}$ of $v$ with respect to the axis of $\varphi$ is also a vertex of $U$. Moreover, $v v^{\prime}$ will be an edge of the $\varphi$-invariant triangulation of $U$, and $v v^{\prime} \varphi(v) \varphi\left(v^{\prime}\right)$ will be one of its ideal tetrahedra.

Let $m \in \mathbb{P}^{1} \mathbb{Q}$ be the Farey vertex $(l / 2) / k$ (reduced fraction). We have $m \notin\{\infty, 0, \pm 1\}$ : indeed, $\infty$ is ruled out because $m$ has odd denominator $k$ (coprime to $l$ ); the other possibilities are ruled out because we assumed $\pm(k, l) \notin\{(1,0),(1, \pm 2)\}$. According to the value of $m$, choose $(p, q, r)$ as in Section 5.2, with the four extra possibilities

$$
\begin{array}{r||c|c|c|c}
\text { if } & m=-2 & m=-1 / 2 & m=1 / 2 & m=2 \\
\hline(p, q, r)= & (\infty,-1,0) & (0,-1, \infty) & (0,1, \infty) & (\infty, 1,0)
\end{array}
$$

(in fact we may switch $p, q$ in these four cases). One then has $m \wedge r \geq 2$. Note that, unlike in Section 2, $m$ is now allowed to be the common Farey neighbor of $p$ and $q$ opposite $r$.

Below we describe an ideal triangulation $\mathcal{D}$ for a solid torus $Y$ (with two ideal points); Proposition 29 will then be the analogue for $\mathcal{D}$ of Proposition 10. For convenience, we 
will first describe a family of tetrahedra of $\mathbb{H}^{3}$ whose vertices are points of $\mathbb{Z}[\sqrt{-1}] \subset$ $\mathbb{P}^{1} \mathbb{C} \simeq \partial_{\infty} \mathbb{H}^{3}$, then only remember the combinatorics of the gluing of these tetrahedra.

The sequence of Farey triangles crossed by the oriented line $\ell$ from $r$ to $m$ is $p q r=$ $T_{0}, T_{1}, \ldots, T_{N}=m s t$ (for some Farey vertices $s, t$, and with $N \geq 1$ - note that in Section 2 we had $N \geq 2$ ). For every index $0 \leq i \leq N$, let $x_{i}, y_{i}, z_{i} \in \mathbb{P}^{1} \mathbb{Q}$ be the vertices of $T_{i}$. Consider the triangulation $\mathcal{T}_{i}$ of $\mathbb{C}$ with vertex set $\mathbb{Z}[\sqrt{-1}]$ and whose edges are precisely all segments of slopes $x_{i}, y_{i}, z_{i}$ between points of $\mathbb{Z}[\sqrt{-1}]$. Each triangle of $\mathcal{T}_{i}$ is the vertical projection of an ideal triangle of $\mathbb{H}^{3}$ with the same triple of vertices. The union of all these ideal triangles, modulo $G:=2 \mathbb{Z} \oplus \sqrt{-1} \mathbb{Z}$, is a twice-punctured torus $\tau_{i}$ in $\mathbb{H}^{3} / G$. If $0<i \leq N$ then the space between $\tau_{i-1}$ and $\tau_{i}$ is the union of two ideal tetrahedra $\dot{\Delta}_{i}$ and $\ddot{\Delta}_{i}$ (glued together along some of their edges). Note that the index $i=N$ is now allowed, unlike in Section 2, so that eg the tetrahedron $\dot{\Delta}_{N}$ (belonging to the last pair) has an edge of slope $m$, the meridian. Also note that since $m=(l / 2) / k$ and $k+(l / 2) \sqrt{-1} \notin G$ (because $k$ is odd), this edge of slope $m$ runs from one of the punctures of $\tau_{N}$ (or $\tau_{0}$ ) to the other.

Consider now the triangulation $\left\{\dot{\Delta}_{i}, \ddot{\Delta}_{i}\right\}_{1 \leq i \leq N}$ as a combinatorial object only. To "kill" the slope $m$, we identify the edges of slope $m$ in $\dot{\Delta}_{N}$ and $\ddot{\Delta}_{N}$, and fill the remaining space with a single tetrahedron $\Delta_{N+1}$ all of whose four faces are glued to the inner faces of $\dot{\Delta}_{N} \cup \ddot{\Delta}_{N}$. This $\Delta_{N+1}$ is the tetrahedron referred to as " $v v^{\prime} \varphi(v) \varphi\left(v^{\prime}\right)$ " at the beginning of Section 5.3. We denote by $\mathcal{D}$ the triangulation $\bigcup_{i=1}^{N}\left\{\dot{\Delta}_{i}, \ddot{\Delta}_{i}\right\} \cup\left\{\Delta_{N+1}\right\}$ and by $Y$ its underlying space, a twice-punctured solid torus. Note that $\mathcal{D}$ admits a combinatorial involution $\iota$ exchanging $\dot{\Delta}_{i}$ with $\ddot{\Delta}_{i}$ for all $1 \leq i \leq N$ (and fixing $\Delta_{N+1}$ setwise): this $\iota$ extends the translation of $\partial Y$ that shifts one puncture to the other.

The ideal link of each of the two ideal vertices of $Y$ (which are exchanged by $\iota$ ) consists of nested hexagons as in Figure 6, but the innermost hexagon is now $H_{N}$ (not $H_{N-1}$ ), and is not collapsed to a broken line of three segments. Instead, the effect of identifying the edges of slope $m$ has been to identify a pair of opposite vertices of $H_{N}$ (namely the inward-pointing vertices); the inside of $H_{N}$ is the union of two triangles joined by a vertex. These two triangles are two vertex links of the tetrahedron $\Delta_{N+1}$ (the other two are in the other ideal vertex of $Y$ ). See Figure 12.

We will not consider the full space of angle structures for our triangulation $\mathcal{D}$ of $M_{s}$ : rather, we will restrict to $\iota$-invariant angle structures (ie angle structures in which for each $1 \leq i \leq N$, the tetrahedra $\dot{\Delta}_{i}$ and $\ddot{\Delta}_{i}$ have the same dihedral angles). Note that if there is an angle structure, we can always average it with its push-forward by $\iota$ to get an $\iota$-invariant angle structure. 


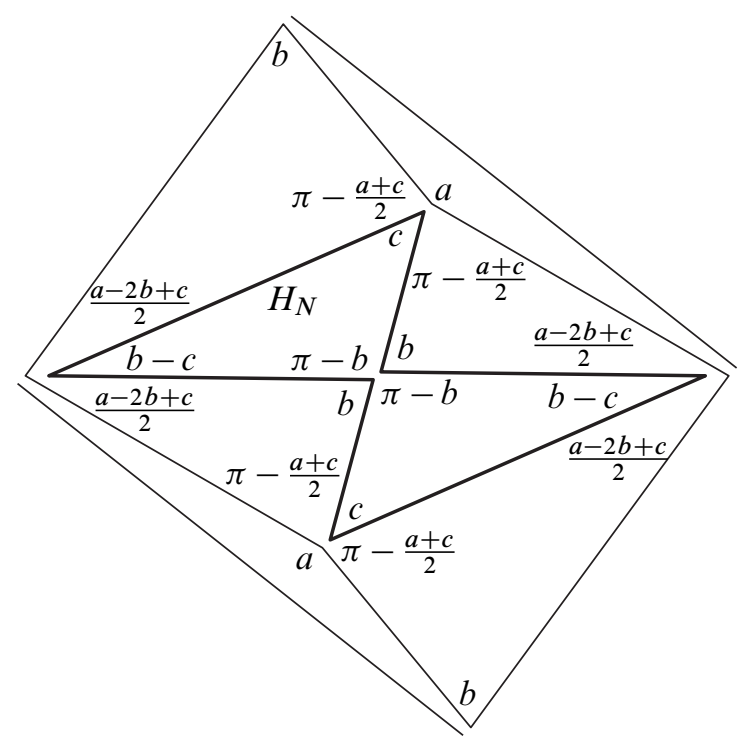

Figure 12: The innermost hexagon $H_{N}$ along with $H_{N-1}$ and the links (Euclidean triangles) of the tetrahedra $\Delta_{N+1}, \dot{\Delta}_{N}, \ddot{\Delta}_{N}$. The angles around each interior vertex sum to $2 \pi$.

Proposition 29 Consider nonnegative reals $\theta_{p}, \theta_{q}, \theta_{r}$ which satisfy (5), namely $0<\theta_{r}<\pi=\theta_{p}+\theta_{q}+\theta_{r}$. The space of $\iota$-invariant angle structures on $\mathcal{D}$ that induce exterior dihedral angles $\theta_{p}, \theta_{q}, \theta_{r}$ at the edges of slope $p, q, r$ of $\partial Y$ (also called $\left(\theta_{p}, \theta_{q}, \theta_{r}\right)$-angle structures) is nonempty.

Remark 30 Proposition 29 requires no inequality like Proposition 10, but that is only because "problematic" slopes $(k, l)$ have already been ruled out.

Proof As in Section 2.3, we introduce an angle parameter $z_{i} \in(0, \pi)$ for every pair of ideal tetrahedra $\dot{\Delta}_{i}, \ddot{\Delta}_{i}$ (where $1 \leq i \leq N$ ). In what follows, $\dot{\Delta}_{i}$ and $\ddot{\Delta}_{i}$ will always be assumed to have the same dihedral angles (they are exchanged by the combinatorial symmetry $\iota$ ). We also denote by $z_{N+1}$ the dihedral angle of $\Delta_{N+1}$ at the edge whose slope is the only rational (Farey vertex) in $T_{N} \cap T_{N-1} \backslash T_{N-2}$. Using these conventions and writing $(a, b, c):=\left(z_{N-1}, z_{N}, z_{N+1}\right)$, it is easy to see that the triples of dihedral angles of the ideal tetrahedra are as follows:

$$
\begin{gathered}
\dot{\Delta}_{N}, \ddot{\Delta}_{N}:\left(b, \pi-\frac{a+c}{2}, \frac{a-2 b+c}{2}\right) \\
\Delta_{N+1}:(c, \quad \pi-b, \quad b-c)
\end{gathered}
$$

(see also Figure 12). For $1 \leq i<N$, the dihedral angles of $\dot{\Delta}_{i}, \ddot{\Delta}_{i}$ are simply given by Table (9). In keeping with Table (9), we consider $z_{N}$ to be a nonhinge parameter. 
Recall that $N \geq 1$ : analogously to (7)-(10), we are thus looking for sequences of the form

$$
\begin{aligned}
& \left(z_{0}, \quad z_{1}, z_{2}, \ldots, z_{N}, z_{N+1}\right) \\
& =\left(\pi+\theta_{q}, \pi-\theta_{r}, z_{2}, \ldots, z_{N}, z_{N+1}\right)
\end{aligned}
$$

subject to the conditions

$$
\left\{\begin{array}{lll}
z_{i-1}>z_{i}+z_{i+1} & \text { if } z_{i} \text { is a hinge parameter } & \text { (hinge condition); } \\
z_{i-1}+z_{i+1}>2 z_{i} & \text { if not } & \text { (convexity condition), eg } i=1 \text { or } N \\
0<z_{i}<\pi & \text { for all } 2 \leq i \leq N & \text { (range condition); } \\
0<z_{2}<\pi-\theta_{q} & \text { as in (10) above; } \\
0<z_{N+1}<z_{N} & \text { which follows from (19). }
\end{array}\right.
$$

To find such a sequence, the argument that finishes Section 2.3 follows through essentially unchanged: we construct a convex positive decreasing sequence $\left(z_{i}\right)_{0 \leq i \leq h}$ where $h$ is the smallest hinge index (or $h=N+1$ if there are no hinges), then set eg $z_{i+1}=\varepsilon z_{i}$ (inductively) for all $i \geq h$ and a fixed small $\varepsilon>0$.

Finally, we must glue the faces of the solid torus $Y$ together to form the Dehn filling $M_{S}$ of the Whitehead link complement. This is performed exactly as in Section 5.2: we set $\left(\theta_{p}, \theta_{q}, \theta_{r}\right)$ as in (18) for $0<\theta<\pi$, so that the faces of $\partial Y$ become two ideal quadrilaterals $P, P^{\prime}$ with edges of slopes 0 and $\infty$; then glue $P$ to $P^{\prime}$ by an orientationreversing homeomorphism sending the edges of slope 0 of $P$ to the edges of slope $\infty$ of $P^{\prime}$ (and conversely). The angular gluing equations are automatically satisfied.

Therefore, the full space $W$ of $\iota$-invariant angle structures for $\mathcal{D}$ is obtained by letting $\theta$ range over $(0, \pi)$ and finding all $\left(\theta_{p}, \theta_{q}, \theta_{r}\right)$-angle structures in the sense of Proposition 29.

Proposition 31 The volume functional has a critical point, namely a maximum, on $W$.

Proof As in Proposition 15, the maximum of the (extended) volume functional is achieved at some point $z$ of the closure $\bar{W}$ of $W$. Using (18), the system of constraints (7) becomes

or

$$
\begin{aligned}
& \left(z_{0}, z_{1}, z_{2}, \ldots, z_{N}, z_{N+1}\right) \\
& =\left(\pi, \theta, z_{2}, \ldots, z_{N}, z_{N+1}\right) \\
& \left(\pi+\theta, \theta, z_{2}, \ldots, z_{N}, z_{N+1}\right)
\end{aligned}
$$

according to the value of $m$. 
The assumption $\theta=\pi$ leads to a contradiction exactly as in the proof of Proposition 28. Therefore $\theta<\pi$.

By (19), $\dot{\Delta}_{N}$ and $\ddot{\Delta}_{N}$ have a dihedral angle equal to $b:=z_{N}$, while $\Delta_{N+1}$ has an angle $\pi-b$. On the other hand, a tetrahedron of $\mathcal{D}$ is flat at $z \in \bar{W}$ if and only if one (and therefore all) of its angles belong to $\{0, \pi\}$ (Proposition 7). Thus, $\dot{\Delta}_{N}, \ddot{\Delta}_{N}$ are flat if and only if $\Delta_{N+1}$ is flat (ie $b \in\{0, \pi\}$ ). The argument of Proposition 15 then follows through: at $z$, if some tetrahedra were flat, all would be flat and the volume would be 0 ; absurd. Thus $z \in W$.

To apply Theorem 5, we only need to make sure that the critical point (maximum) of $\mathcal{V}$ on the space $W$ of $\iota$-invariant angle structures is also critical (maximal) in the space of all angle structures: but that is clear since by concavity of the volume functional (Fact 6), the volume can only go up when we average an angle structure with its push-forward by $\iota$. Theorem 5 does apply: we have found a complete hyperbolic structure on the triangulated space $M_{s}$. To check that the triangulation is canonical, we only need to check the Minkowski convexity relationship (12). For boundary faces of $Y$, the situation is exactly the same as in Case 1 (odd $l$ ). For interior faces of $Y$ not bounding the "extra" tetrahedron $\Delta_{N+1}$, we proceed as in Section 4.4: the only new argument needed is an analogue of Proposition 23 (predicting the handednesses of powers of the core curve of $Y$ ), namely:

Proposition 32 Let $T_{i}$ be a Farey triangle such that $0<i<N$ and let $x \in \mathbb{P}^{1} \mathbb{Q}$ be the Farey vertex $T_{i-1} \cap T_{i} \cap T_{i+1}$. Consider a properly embedded line $\gamma_{x}$ of slope $x$ in $\partial Y$ (running between two cusps), and a lift $\widehat{\gamma_{x}}$ of $\gamma_{x}$ to a universal cover $U \subset \mathbb{H}^{3}$ of $Y$ (running between two ideal points). The deck transformation of $U$ that sends the initial point of $\widehat{\gamma_{x}}$ to the final point is left-handed (resp. right-handed) if and only if the Farey triangle $T_{i}$ carries a letter $L$ (resp. $R$ ).

Proof The proof is exactly as in Section 3. The key argument that the integral $\lambda_{x}$ of the longitude 1 -form along $\widehat{\gamma_{x}}$ stays less than $\pi$ is only easier, because the "longest" curve $\gamma_{m}$ runs only around one half, not all, of the meridian of $U$ (connecting some ideal vertex to its symmetric image with respect to the axis of $U$ ); thus $\lambda_{m}=\pi$ and $\lambda_{x}<\pi$.

The only remaining case of the Minkowski convexity relationship (12) is at the faces of $\Delta_{N+1}$. According to our picture of the cusp triangulation (Figure 12), we can assume that the innermost hexagon $H_{N}$ has vertices at

$-1, \quad 0, \quad \zeta, \quad 1, \quad 0, \quad-\zeta$ 
and look at the interface $\zeta \infty 0$ between ideal tetrahedra $1 \zeta \infty 0$ and $-1 \zeta \infty 0$.

Following the method of Section 4.4, if $\zeta=\xi+i \eta$, the isotropic vectors in $\mathbb{R}^{3+1}$ corresponding to the horoballs centered at $\infty, 0, \zeta, 1,-1$ are respectively

$$
\begin{aligned}
& v_{\infty}=\quad\left(\begin{array}{lllll}
0, & 0, & -1, & 1
\end{array}\right) \\
& v_{0}=\frac{1}{|\zeta|}\left(\begin{array}{llll}
0, & 0, & 1, & 1
\end{array}\right) \\
& v_{\zeta}=\frac{1}{|\zeta||\zeta-1|}\left(2 \xi, 2 \eta, 1-|\zeta|^{2}, 1+|\zeta|^{2}\right) \\
& v_{1}=\frac{1}{|\zeta-1|}\left(\begin{array}{llll}
2, & 0, & 0, & 2
\end{array}\right) \\
& v_{-1}=\frac{1}{|\zeta-1|}\left(\begin{array}{lllll}
-2, & 0, & 0, & 2
\end{array}\right) \text {. }
\end{aligned}
$$

The solution to $\rho v_{1}+(1-\rho) v_{-1}=\lambda v_{\infty}+\mu v_{0}+v v_{\zeta}$ satisfies

$$
(\lambda, \mu, v)=\left(\frac{1}{|\zeta-1|}, \frac{|\zeta|}{|\zeta-1|}, 0\right), \quad \text { hence } \lambda+\mu+v=\frac{|\zeta|+1}{|\zeta-1|}>1
$$

according to the triangular inequality in the triangle $(0,1, \zeta)$ : by Proposition 25 , the convexity inequality in Minkowski space is satisfied.

\subsection{Delaunay decompositions and elementary Kleinian groups}

Remark 33 If $U \subset \mathbb{H}^{3}$ is a (triangulated) universal cover of the solid torus $Y$ and $\langle\varphi\rangle$ is the group of deck transformations of $U$, we mentioned at the beginning of Section 5.3 that for each ideal vertex $v$ of $U$, the symmetric image $v^{\prime}$ of $v$ with respect to the axis of $\varphi$ is also a vertex of $U$, and $\Delta:=v v^{\prime} \varphi(v) \varphi\left(v^{\prime}\right)$ is an ideal tetrahedron of $U$ (projecting to $\Delta_{N+1}$ ). By duality between the Ford-Voronoi domain and the canonical triangulation, the last computation of Section 5.3 amounts to checking the following (easy) fact: if all vertices of $U$ are endowed with horoballs of the same size, then the center of $\Delta$ is nearer to the horoballs centered at the vertices of $\Delta$ than to any other horoballs.

More generally, if $n \geq 3$, let $G:=\langle\varphi, \psi\rangle \subset \operatorname{Isom}^{+}\left(\mathbb{H}^{3}\right)$ be an elementary group generated by a loxodromy $\varphi$ and an order $-n$ rotation $\psi$ with the same axis $\delta$ (note that Section 5.3 amounted to the case $n=2$, and Section 2 to the case $n=1$ ). Let $\mathcal{O}:=G p \subset \partial_{\infty} \mathbb{H}^{3}$ be a generic ideal orbit of $G$; if $h_{p}$ is a horoball centered at $p$, all horoballs in the $G$-orbit of $h_{p}$ come equally close to the line $\delta$. The convex hull of $\mathcal{O}$ projects modulo $\varphi$ to an $n$-times punctured solid torus $X$ whose boundary is pleated along a certain ideal triangulation in which all vertices have the same degree (generically 6 , exceptionally 4 ; for simplicity let us assume the generic situation). The convex hull construction in Minkowski space $\mathbb{R}^{3+1}$ yields a decomposition of $X$ 
into ideal polyhedra with respect to the horoballs $G h_{p}$. The central polyhedron is the convex hull $Q$ of $\langle\psi\rangle p \cup \varphi(\langle\psi\rangle p)$, namely an ideal hyperbolic antiprism with regular $n$-sided bases (glued together via $\varphi$ ): indeed, it is easy to check that the center of $Q$ is closer to the horoballs centered at the vertices of $Q$ than to any other horoballs of the $G$-orbit.

It is possible that $Q$ is the only cell of $X$. Otherwise, we claim that the remaining cells between $Q$ and $\partial X$ are tetrahedra glued together according to diagonal exchanges and Farey-type combinatorics: namely, $\partial X / \psi$ is a once-punctured torus with ideal edges of slope $p, q, r \in \mathbb{P}^{1} \mathbb{Q}$ for some arbitrary marking (these slopes are mutual Farey neighbors). The meridian of $\mathrm{X}$ defines the $n$-th power of an irreducible element of $H_{1}(\partial X / \psi, \mathbb{Z})$, and therefore also a slope $m \in \mathbb{P}^{1} \mathbb{Q}$. Since $m$ is the slope of the base edges of the antiprism $Q$, if $Q$ is the only cell in $X$ then $m \in\{p, q, r\}$. Otherwise, we may as in Section 2 and Section 5.3 assume that the Farey edge $p q$ separates $m$ from $r$ and follow the line $\ell$ from $r$ to $m$ to construct a (combinatorial) ideal decomposition $\mathcal{D}$ of $X$.

In fact, the following "Gauss-Bonnet type" result (left as an exercise) is a simple generalization of the method worked out in this paper. It uses the fact that the antiprism $Q$ (like any convex ideal hyperbolic polyhedron - see Rivin [24] and Guéritaud [13]) is uniquely determined up to isometry by its dihedral angles.

Theorem 34 Consider nonnegative reals $\theta_{p}, \theta_{q}, \theta_{r}$ satisfying (5), namely $0<\theta_{r}<$ $\pi=\theta_{p}+\theta_{q}+\theta_{r}$. There exists a hyperbolic $n$-times punctured solid torus $X$, decomposed into convex ideal polyhedra according to the combinatorics of $\mathcal{D}$ and with exterior dihedral angles $\theta_{p}, \theta_{q}, \theta_{r}$ at the edges of slope $p, q, r$, if and only if

$$
(m \wedge p) \theta_{p}+(m \wedge q) \theta_{q}+(m \wedge r) \theta_{r}>\frac{2 \pi}{n} .
$$

Moreover, $X$ is then unique up to isometry and $\mathcal{D}$ is the Delaunay decomposition of $X$.

\section{References}

[1] H Akiyoshi, On the hyperbolic manifolds obtained from the Whitehead link, from: "Analysis of discrete groups, II (Kyoto, 1996)", Sūrikaisekikenkyūsho Kōkyūroku 1022 (1997) 213-224 MR1643724

[2] H Akiyoshi, On the Ford domains of once-punctured torus groups, from: "Hyperbolic spaces and related topics (Japanese) (Kyoto, 1998)", Sūrikaisekikenkyūsho Kōkyūroku 1104 (1999) 109-121 MR1744475 
[3] H Akiyoshi, Finiteness of polyhedral decompositions of cusped hyperbolic manifolds obtained by the Epstein-Penner's method, Proc. Amer. Math. Soc. 129 (2001) 24312439 MR1823928

[4] H Akiyoshi, Canonical decompositions of cusped hyperbolic 3-manifolds obtained by Dehn filling, from: "Perspectives of hyperbolic spaces", Kokyuroku 1329, RIMS, Kyoto (2003) 121-132

[5] H Akiyoshi, M Sakuma, Comparing two convex hull constructions for cusped hyperbolic manifolds, from: "Kleinian groups and hyperbolic 3-manifolds (Warwick, 2001)", (Y Komori, V Markovic, C Series, editors), London Math. Soc. Lecture Note Ser. 299, Cambridge Univ. Press (2003) 209-246 MR2044552

[6] H Akiyoshi, M Sakuma, M Wada, Y Yamashita, Jørgensen's picture of punctured torus groups and its refinement, from: "Kleinian groups and hyperbolic 3-manifolds (Warwick, 2001)", (Y Komori, V Markovic, C Series, editors), London Math. Soc. Lecture Note Ser. 299, Cambridge Univ. Press (2003) 247-273 MR2044553

[7] H Akiyoshi, M Sakuma, M Wada, Y Yamashita, Punctured torus groups and 2-bridge knot groups. I, Lecture Notes in Math. 1909, Springer, Berlin (2007) MR2330319

[8] P J Callahan, M V Hildebrand, J R Weeks, A census of cusped hyperbolic 3manifolds, Math. Comp. 68 (1999) 321-332 MR1620219

[9] K Chan, Constructing hyperbolic 3-manifolds, Undergraduate thesis with Craig Hodgson, University of Melbourne (2002)

[10] T A Drumm, J A Poritz, Ford and Dirichlet domains for cyclic subgroups of $\mathrm{PSL}_{2}(\mathbf{C})$ acting on $\mathbf{H}_{R}^{3}$ and $\partial \mathbf{H}_{R}^{3}$, Conform. Geom. Dyn. 3 (1999) 116-150 MR1716572

[11] D B A Epstein, R C Penner, Euclidean decompositions of noncompact hyperbolic manifolds, J. Differential Geom. 27 (1988) 67-80 MR918457

[12] D Futer, F Guéritaud, Angled decompositions of arborescent link complements, Proc. Lond. Math. Soc. (3) 98 (2009) 325-364 MR2481951

[13] F Guéritaud, On an elementary proof of Rivin's characterization of convex ideal hyperbolic polyhedra by their dihedral angles, Geom. Dedicata 108 (2004) 111-124 MR2112668

[14] F Guéritaud, Géométrie hyperbolique effective et triangulations idéales canoniques en dimension trois, $\mathrm{PhD}$ thesis, Université d'Orsay (2006)

[15] F Guéritaud, On canonical triangulations of once-punctured torus bundles and twobridge link complements, Geom. Topol. 10 (2006) 1239-1284 MR2255497 With an appendix by D Futer

[16] F Guéritaud, Triangulated cores of punctured-torus groups, J. Differential Geom. 81 (2009) 91-142 MR2477892 
[17] B Jaco, H Rubinstein, Layered-triangulations of 3-manifolds, Preprint (2006) Available at http://www.math.okstate.edu/ jaco/

[18] T Jørgensen, On cyclic groups of Möbius transformations, Math. Scand. 33 (1973) 250-260 (1974) MR0348103

[19] T Jørgensen, On pairs of once-punctured tori, from: "Kleinian groups and hyperbolic 3manifolds (Warwick, 2001)", (Y Komori, V Markovic, C Series, editors), London Math. Soc. Lecture Note Ser. 299, Cambridge Univ. Press (2003) 183-207 MR2044551

[20] M Lackenby, The canonical decomposition of once-punctured torus bundles, Comment. Math. Helv. 78 (2003) 363-384 MR1988201

[21] B Martelli, C Petronio, Dehn filling of the "magic" 3-manifold, Comm. Anal. Geom. 14 (2006) 969-1026 MR2287152

[22] W D Neumann, A W Reid, Arithmetic of hyperbolic manifolds, from: "Topology '90 (Columbus, OH, 1990)", (B Apanasov, W D Neumann, A W Reid, L Siebenmann, editors), Ohio State Univ. Math. Res. Inst. Publ. 1, de Gruyter, Berlin (1992) 273-310 MR1184416

[23] I Rivin, Euclidean structures on simplicial surfaces and hyperbolic volume, Ann. of Math. (2) 139 (1994) 553-580 MR1283870

[24] I Rivin, Combinatorial optimization in geometry, Adv. in Appl. Math. 31 (2003) 242271 MR1985831

[25] M Sakuma, J Weeks, Examples of canonical decompositions of hyperbolic link complements, Japan. J. Math. (N.S.) 21 (1995) 393-439 MR1364387

[26] W P Thurston, The geometry and topology of three-manifolds, Princeton Univ. Math. Dept. Lecture Notes (1979) Available at http://msri.org/publications/books/ gt $3 \mathrm{~m} /$

[27] J R Weeks, SnapPea: a software for the study of hyperbolic manifolds Available at http: //www. geometrygames .org/SnapPea/

[28] J R Weeks, Convex hulls and isometries of cusped hyperbolic 3-manifolds, Topology Appl. 52 (1993) 127-149 MR1241189

Laboratoire Paul-Painlevé, CNRS UMR 8524, Université de Lille 1

59650 Villeneuve d'Ascq, France

Mathematics Institute, University of Warwick

Coventry CV4 7AL, UK

Francois.Gueritaud@math.univ-lille1.fr, s.schleimer@warwick.ac.uk http://www.warwick.ac.uk/ masgar/

Proposed: David Gabai

Seconded: Walter Neumann, Joan Birman
Received: 29 July 2008 Revised: 22 September 2009 